\title{
Darboux Transformations for $(2+1)$-Dimensional Extensions of the KP Hierarchy
}

\author{
Oleksandr CHVARTATSKYI ${ }^{\dagger}$ and Yuriy SYDORENKO $\ddagger$ \\ $\dagger$ Mathematisches Institut, Georg-August-Universität Göttingen, 37073 Göttingen, Germany \\ E-mail: alex.chvartatskyy@gmail.com \\ $\ddagger$ Faculty of Mechanics and Mathematics, Ivan Franko National University of Lviv, \\ 79000 Lviv, Ukraine \\ E-mail: y_sydorenko@franko.lviv.ua
}

Received September 23, 2014, in final form March 27, 2015; Published online April 10, 2015

http://dx.doi.org/10.3842/SIGMA.2015.028

\begin{abstract}
New extensions of the KP and modified KP hierarchies with self-consistent sources are proposed. The latter provide new generalizations of $(2+1)$-dimensional integrable equations, including the DS-III equation and the $N$-wave problem. Furthermore, we recover a system that contains two types of the KP equation with self-consistent sources as special cases. Darboux and binary Darboux transformations are applied to generate solutions of the proposed hierarchies.
\end{abstract}

Key words: KP hierarchy; symmetry constraints; binary Darboux transformation; DaveyStewartson equation; KP equation with self-consistent sources

2010 Mathematics Subject Classification: 35Q51; 35Q53; 35Q55; 37K35

\section{Introduction}

In the past years, a lot of attention have been given to the study of Kadomtsev-Petviashvili hierarchy (KP hierarchy) and its generalizations from both physical and mathematical points of view $[1,13,55,57,61,74]$. KP equation with self-consistent sources and related $k$-constrained KP ( $k$-cKP) hierarchy also present an interest $[4,9,10,33,40,42,52,53,63,64,65,78]$. The latter hierarchy contains, in particular, nonlinear Schrödinger equation, Yajima-Oikawa equation, extension of the Boussinesq equation and $\mathrm{KdV}$ equation with self-consistent sources. A modified $k$-constrained KP $(k$-cmKP) hierarchy was proposed in $[8,43,59]$. The $k$-cKP hierarchy was extended to $2+1$ dimensions $((2+1)$-dimensional $k$-cKP hierarchy $)$ in $[48,54,73]$.

A powerful solution generating method for nonlinear integrable systems is based on the Darboux transformations (DT) and the binary Darboux transformations (BDT) [51]. The latter transformations were also applied to $k$-cKP hierarchy and its $(2+1)$-dimensional extensions (see $[58,82]$ and $[47,73]$ respectively). More general $(2+1)$-dimensional extensions of the $k$ cKP hierarchy and the corresponding solutions were investigated in [11]. The latter hierarchies cover matrix generalizations of the Davey-Stewartson (DS) and Nizhnik-Novikov-Veselov (NNV) systems, (2+1)-dimensional extensions of the Yajima-Oikawa and modified Korteweg-de Vries equations.

Hamiltonian analysis for the above mentioned hierarchies, which is based on group-theoretical and Lie-algebraic methods, was elaborated in [5, 31, 34, 68, 69, 70]. Analytical scheme of the Hamiltonian analysis was described in [28].

The main aim of this work is to present new $(2+1)$-dimensional extensions of $k$-cKP and modified $k$-cKP hierarchies. It is organized as follows. In Section 2 we consider reductions (2.2) of Lax operators $L_{k}$ and $M_{n}$ involving nonzero integral terms with degenerate kernels. The latter reductions allow us to obtain a hierarchy that is more general then $(2+1)$-dimensional 
extensions of the $k$-cKP hierarchy that we considered in [11]. This is shown in Remark 2.5 (Section 2), which describes important special cases of the obtained hierarchy. KP hierarchy as a special case is also included. In Section 2 we also list some nonlinear integrable systems that are provided by Lax pairs (2.2). In particular, we get new generalizations of the $N$-wave problem, the matrix Davey-Stewartson (DS-III) equation and the matrix KP equation with self-consistent sources (KPSCS). Despite the fact that the latter systems have more compact scalar counterparts, we also present their matrix versions due to the recent interest in matrix and, more generally, noncommutative integrable systems (see, e.g., [14, 15, 32, 44, 71, 72, 75]).

In Section 3 we present a solution generating technique (dressing method) for hierarchies (2.2), (2.6) using DTs and BDTs. New $(2+1)$-dimensional extensions of the modified $k$-cKP hierarchies and corresponding solution generating methods are discussed in Section 4. Some matrix integrable equations from the respective hierarchy are also listed. This includes new extensions of the matrix Chen-Lee-Liu equation and the modified KP equation with self-consistent sources. A short summary of the obtained results and some problems for future investigation are presented in Conclusions.

\section{New $(2+1)$-dimensional generalizations of the $k$-constrained KP hierarchy}

Further we will use the calculus of the integro-differential (pseudo-differential) operators of the form $L=\sum_{i=-\infty}^{l} f_{i} D^{i}, l \in \mathbb{Z}$ (see, e.g., [13]). Coefficients $f_{i}, i \in \mathbb{Z}$, are matrix-valued functions and symbol $D:=\frac{\partial}{\partial x}$ denotes the derivative with respect to $x$. Composition (multiplication) of integro-differential operators is generated by the commutation rule:

$$
D^{n} f:=\sum_{j=0}^{\infty}\left(\begin{array}{c}
n \\
j
\end{array}\right) f^{(j)} D^{n-j}, \quad f^{(j)}:=\frac{\partial^{j} f}{\partial x^{j}}, \quad n \in \mathbb{Z},
$$

where $\left(\begin{array}{c}n \\ 0\end{array}\right):=1,\left(\begin{array}{c}n \\ j\end{array}\right):=\frac{n(n-1) \cdots(n-j+1)}{j !} . D^{n} f$ stands for the composition of the operator $D^{n}$ and the operator of multiplication by matrix-valued function $f$, whereas by curly brackets we will denote the action of the operator on the function, i.e., $D^{n}\{f\}:=f^{(n)}=\frac{\partial^{n} f}{\partial x^{n}}$. More generally, we will use notations $L\{f\}$ and $L f$ in the same manner.

Consider Sato-Zakharov-Shabat dressing operator:

$$
W=I+w_{1} D^{-1}+w_{2} D^{-2}+\cdots
$$

with $(N \times N)$-matrix-valued coefficients $w_{i}$. Introduce two differential operators $\beta_{k} \partial_{\tau_{k}}-\mathcal{J}_{k} D^{k}$ and $\alpha_{n} \partial_{t_{n}}-\tilde{\mathcal{J}}_{n} D^{n}, \alpha_{n}, \beta_{k} \in \mathbb{C}, n, k \in \mathbb{N}$, where $\mathcal{J}_{k}$ and $\tilde{\mathcal{J}}_{n}$ are $N \times N$ commuting matrices (i.e., $\left.\left[\tilde{\mathcal{J}}_{n}, \mathcal{J}_{k}\right]=0\right)$. It is evident that dressed operators have the form:

$$
\begin{aligned}
& L_{k}:=W\left(\beta_{k} \partial_{\tau_{k}}-\mathcal{J}_{k} D^{k}\right) W^{-1}=\beta_{k} \partial_{\tau_{k}}-B_{k}-u_{-1} D^{-1}-u_{-2} D^{-2}-\cdots, \\
& M_{n}:=W\left(\alpha_{n} \partial_{t_{n}}-\tilde{\mathcal{J}}_{n} D^{n}\right) W^{-1}=\alpha_{n} \partial_{t_{n}}-A_{n}-v_{-1} D^{-1}-v_{-2} D^{-2}-\cdots, \\
& B_{k}=\sum_{j=0}^{k} u_{j} D^{j}, \quad u_{k}=\mathcal{J}_{k}, \quad A_{n}=\sum_{i=0}^{n} v_{i} D^{i}, \quad v_{n}=\tilde{\mathcal{J}}_{n},
\end{aligned}
$$

where $u_{j}$ and $v_{i}$ are matrix-valued functions of dimension $N \times N$. Impose the following reduction on the integral parts of operators $L_{k}$ and $M_{n}$ :

$$
L_{k}=\beta_{k} \partial_{\tau_{k}}-B_{k}-\mathbf{q} \mathcal{M}_{0} D^{-1} \mathbf{r}^{\top}, \quad M_{n}=\alpha_{n} \partial_{t_{n}}-A_{n}-\tilde{\mathbf{q}} \tilde{\mathcal{M}}_{0} D^{-1} \tilde{\mathbf{r}}^{\top},
$$


where $\mathbf{q}$ and $\mathbf{r}$ are matrix-valued functions with dimension $N \times m ; \tilde{\mathbf{q}}$ and $\tilde{\mathbf{r}}$ are matrix-valued functions with dimension $N \times \tilde{m} . \mathcal{M}_{0}$ and $\tilde{\mathcal{M}}_{0}$ are constant matrices with dimensions $m \times m$ and $\tilde{m} \times \tilde{m}$ respectively.

Reductions (2.2) generalize the corresponding analogues obtained in [11]. It will be shown at the end of this section (reductions (2.11) and Remark 2.5). Moreover, Lax pairs given by $L_{k}$ and $M_{n}(2.2)$ remain covariant under the action of Darboux and Binary Darboux Transformations (see Section 3), which allows to construct families of solutions for the corresponding integrable systems. Lax pairs $(2.2)$ can be also considered as matrix $(2+1)$-dimensional generalizations of the respective operators appearing in the study of dispersive analogues of Benny's equations [25].

For technical purposes we will use the following statement:

Proposition 2.1. For matrix-valued functions $h_{1}$ and $h_{2}$ and differential operator $A=\sum_{i=0}^{l} f_{i} D^{i}$, $l \in \mathbb{N}$, with matrix-valued coefficients $f_{i}$ the following formulae hold:

$$
\begin{aligned}
& A h_{1} D^{-1} h_{2}=\left(A h_{1} D^{-1} h_{2}\right)_{\geq 0}+A\left\{h_{1}\right\} D^{-1} h_{2}, \\
& h_{1} D^{-1} h_{2} A=\left(h_{1} D^{-1} h_{2} A\right)_{\geq 0}+h_{1} D^{-1}\left[A^{\tau}\left\{h_{2}^{\top}\right\}\right]^{\top}, \\
& D^{-1} h_{1} h_{2} D^{-1}=D^{-1}\left\{h_{1} h_{2}\right\} D^{-1}-D^{-1} D^{-1}\left\{h_{1} h_{2}\right\} .
\end{aligned}
$$

Symbol ${ }^{\top}$ in the latter stands for the matrix transpose and $A^{\tau}$ denotes the transpose of $A$, i.e., $A^{\tau}:=\sum_{i=0}^{l}(-1)^{i} D^{i} f_{i}^{\top}$. Subscript $\geq 0$ denotes the differential part of the respective operator (e.g., $\left.\left(\sum_{i=-\infty}^{l} f_{i} D^{i}\right)_{\geq 0}=\sum_{i=0}^{l} f_{i} D^{i}\right)$.

Proof. All three formulae are consequences of the commutation rule (2.1). Let us check (2.4) and (2.5). It is enough to prove (2.4) for $A=f_{l} D^{l}$. Using (2.1) we get

$$
\begin{aligned}
& h_{1} D^{-1} h_{2} A-h_{1} D^{-1}\left[A^{\tau}\left\{h_{2}^{\top}\right\}\right]^{\top}=h_{1} D^{-1} h_{2} f_{l} D^{l}-h_{1} D^{-1}(-1)^{n}\left(h_{2} f_{l}\right)^{(l)} \\
& =h_{1} \sum_{i=0}^{\infty}(-1)^{i}\left(h_{2} f_{l}\right)^{(i)} D^{l-i-1}-h_{1} \sum_{j=0}^{\infty}(-1)^{j+l}\left(h_{2} u_{l}\right)^{(j+l)} D^{-1-j} \\
& =h_{1} \sum_{i=0}^{l-1}(-1)^{i}\left(h_{2} f_{l}\right)^{(i)} D^{l-i-1}=\left(h_{1} D^{-1} h_{2} A\right)_{\geq 0} .
\end{aligned}
$$

(2.5) follows from the following computations:

$$
\begin{aligned}
D^{-1} h_{1} h_{2} D^{-1}+D^{-1} D^{-1}\left\{h_{1} h_{2}\right\} & =\sum_{i=0}^{\infty}(-1)^{i}\left(h_{1} h_{2}\right)^{(i)} D^{-i-2} \\
+ & \sum_{j=0}^{\infty}(-1)^{j}\left(h_{1} h_{2}\right)^{(j-1)} D^{-j-1}=\left(h_{1} h_{2}\right)^{(-1)} D^{-1}=D^{-1}\left\{h_{1} h_{2}\right\} D^{-1} .
\end{aligned}
$$

Remark 2.2. Formulae (2.3)-(2.5) can be found in [11, 79]. Since the respective references do not contain the proof, we decided to present it in order to make the paper self-contained.

The following statement follows from Proposition 2.1.

Proposition 2.3. Assume that the following equations hold:

$$
L_{k}\{\tilde{\mathbf{q}}\}=\tilde{\mathbf{q}} \Lambda_{\tilde{\mathbf{q}}}, \quad L_{k}^{\tau}\{\tilde{\mathbf{r}}\}=\tilde{\mathbf{r}} \Lambda_{\tilde{\mathbf{r}}}, \quad M_{n}\{\mathbf{q}\}=\mathbf{q} \Lambda_{\mathbf{q}}, \quad M_{n}^{\tau}\{\mathbf{r}\}=\mathbf{r} \Lambda_{\mathbf{r}},
$$


where $\Lambda_{\mathbf{q}}, \Lambda_{\mathbf{r}}$ and $\Lambda_{\tilde{\mathbf{q}}}, \Lambda_{\tilde{\mathbf{r}}}$ are constant matrices with dimensions $(m \times m)$ and $(\tilde{m} \times \tilde{m})$ respectively, that satisfy equations: $\Lambda_{\tilde{\mathbf{q}}} \tilde{\mathcal{M}}_{0}-\tilde{\mathcal{M}}_{0} \Lambda_{\tilde{\mathbf{r}}}^{\top}=0, \Lambda_{\mathbf{q}} \mathcal{M}_{0}-\mathcal{M}_{0} \Lambda_{\mathbf{r}}^{\top}=0$.

Then Lax equation $\left[L_{k}, M_{n}\right]=0$ holds if and only if equation $\left[L_{k}, M_{n}\right]_{\geq 0}=0$ is satisfied.

Proof. From the equality $\left[L_{k}, M_{n}\right]=\left[L_{k}, M_{n}\right]_{\geq 0}+\left[L_{k}, M_{n}\right]_{<0}$ we obtain that Lax equation $\left[L_{k}, M_{n}\right]=0$ is equivalent to the following one:

$$
\left[L_{k}, M_{n}\right]_{\geq 0}=0, \quad\left[L_{k}, M_{n}\right]_{<0}=0 .
$$

Thus, it is sufficient to prove that equalities $L_{k}\{\tilde{\mathbf{q}}\}=\tilde{\mathbf{q}} \Lambda_{\tilde{\mathbf{q}}}, L_{k}^{\tau}\{\tilde{\mathbf{r}}\}=\tilde{\mathbf{r}} \Lambda_{\tilde{\mathbf{r}}}, M_{n}\{\mathbf{q}\}=\mathbf{q} \Lambda_{\mathbf{q}}$ $M_{n}^{\tau}\{\mathbf{r}\}=\mathbf{r} \Lambda_{\mathbf{r}}$ imply $\left[L_{k}, M_{n}\right]_{<0}=0$. From the form of operators $L_{k}, M_{n}$ (2.2) we obtain:

$$
\begin{aligned}
{\left[L_{k}, M_{n}\right]_{<0}=} & {\left[\tilde{\mathbf{q}} \tilde{\mathcal{M}}_{0} D^{-1} \tilde{\mathbf{r}}^{\top}, \beta_{k} \partial_{\tau_{k}}-B_{k}\right]_{<0} } \\
& +\left[\mathbf{q} \mathcal{M}_{0} D^{-1} \mathbf{r}^{\top}, \tilde{\mathbf{q}} \tilde{\mathcal{M}}_{0} D^{-1} \tilde{\mathbf{r}}^{\top}\right]_{<0}+\left[\alpha_{n} \partial_{t_{n}}-A_{n}, \mathbf{q} \mathcal{M}_{0} D^{-1} \mathbf{r}^{\top}\right]_{<0} .
\end{aligned}
$$

After direct computations for each of the three items at the right-hand side of formula (2.7) we get:

$$
\begin{aligned}
& {\left[\tilde{\mathbf{q}} \tilde{\mathcal{M}}_{0} D^{-1} \tilde{\mathbf{r}}^{\top}, \beta_{k} \partial_{\tau_{k}}-B_{k}\right]_{<0}=-\left(\beta_{k} \tilde{\mathbf{q}}_{\tau_{k}}-B_{k}\{\tilde{\mathbf{q}}\}\right) \tilde{\mathcal{M}}_{0} D^{-1} \tilde{\mathbf{r}}^{\top}} \\
& \quad-\tilde{\mathbf{q}} \tilde{\mathcal{M}}_{0} D^{-1}\left(\beta_{k} \tilde{\mathbf{r}}_{\tau_{k}}^{\top}+\left(B_{k}^{\tau}\{\tilde{\mathbf{r}}\}\right)^{\top}\right), \\
& \quad\left[\alpha_{n} \partial_{t_{n}}-A_{n}, \mathbf{q} \mathcal{M}_{0} D^{-1} \mathbf{r}^{\top}\right]_{<0}=\left(\alpha_{n} \mathbf{q}_{t_{n}}-A_{n}\{\mathbf{q}\}\right) \mathcal{M}_{0} D^{-1} \mathbf{r}^{\top} \\
& \quad+\mathbf{q} \mathcal{M}_{0} D^{-1}\left(\alpha_{n} \mathbf{r}_{t_{n}}^{\top}+\left(A_{n}^{\tau}\{\mathbf{r}\}\right)^{\top}\right), \\
& \quad\left[\mathbf{q} \mathcal{M}_{0} D^{-1} \mathbf{r}^{\top}, \tilde{\mathbf{q}} \tilde{\mathcal{M}}_{0} D^{-1} \tilde{\mathbf{r}}^{\top}\right]_{<0}=\mathbf{q} \mathcal{M}_{0} D^{-1}\left\{\mathbf{r}^{\top} \tilde{\mathbf{q}}\right\} \tilde{\mathcal{M}}_{0} D^{-1} \tilde{\mathbf{r}}^{\top}-\mathbf{q} \mathcal{M}_{0} D^{-1} D^{-1}\left\{\mathbf{r}^{\top} \tilde{\mathbf{q}}\right\} \tilde{\mathcal{M}}_{0} \tilde{\mathbf{r}}^{\top} \\
& \quad-\tilde{\mathbf{q}} \tilde{\mathcal{M}}_{0} D^{-1}\left\{\tilde{\mathbf{r}}^{\top} \mathbf{q}\right\} \mathcal{M}_{0} D^{-1} \mathbf{r}^{\top}+\tilde{\mathbf{q}} \tilde{\mathcal{M}}_{0} D^{-1} D^{-1}\left\{\tilde{\mathbf{r}} \tilde{\mathbf{r}}^{\top} \mathbf{q}\right\} \mathcal{M}_{0} \mathbf{r}^{\top} .
\end{aligned}
$$

The latter formulae are consequences of (2.3)-(2.5). From formulae (2.7), (2.8) using (2.6) we get

$$
\begin{aligned}
{\left[L_{k}, M_{n}\right]_{<0}=} & M_{n}\{\mathbf{q}\} \mathcal{M}_{0} D^{-1} \mathbf{r}^{\top}-\mathbf{q} \mathcal{M}_{0} D^{-1}\left(M_{n}^{\tau}\{\mathbf{r}\}\right)^{\top}-L_{k}\{\tilde{\mathbf{q}}\} \tilde{\mathcal{M}}_{0} D^{-1} \tilde{\mathbf{r}}^{\top} \\
& +\tilde{\mathbf{q}} \tilde{\mathcal{M}}_{0} D^{-1}\left(L_{k}^{\tau}\{\tilde{\mathbf{r}}\}\right)^{\top}=\mathbf{q}\left(\Lambda_{\mathbf{q}} \mathcal{M}_{0}-\mathcal{M}_{0} \Lambda_{\mathbf{r}}^{\top}\right) D^{-1} \mathbf{r}^{\top} \\
& -\tilde{\mathbf{q}}\left(\Lambda_{\tilde{\mathbf{q}}} \tilde{\mathcal{M}}_{0}-\tilde{\mathcal{M}}_{0} \Lambda_{\tilde{\mathbf{r}}}^{\top}\right) D^{-1} \tilde{\mathbf{r}}^{\top}=0 .
\end{aligned}
$$

From the last formula we obtain that equality $\left[L_{k}, M_{n}\right]=0$ is equivalent to condition (2.6).

Consider some nonlinear systems that hierarchy given by (2.2) and (2.6) contains. In all examples listed below we assume that equations (2.6) hold. Due to Proposition 2.3 it implies the equivalence of equations $\left[L_{k}, M_{n}\right]=0$ and $\left[L_{k}, M_{n}\right]_{\geq 0}=0$. For simplicity we set $\Lambda_{\mathbf{q}}=\Lambda_{\mathbf{r}}=0$, $\Lambda_{\tilde{\mathbf{q}}}=\Lambda_{\tilde{\mathbf{r}}}=0$.

1. $k=1, n=1$. We shall use the following notation $\beta:=\beta_{1}, \alpha:=\alpha_{1}, \tau:=\tau_{1}, t:=t_{1}$. Then (2.2) reads:

$$
L_{1}=\beta \partial_{\tau}-J D+[J, Q]-\mathbf{q} \mathcal{M}_{0} D^{-1} \mathbf{r}^{\top}, \quad M_{1}=\alpha \partial_{t}-\tilde{J} D+[\tilde{J}, Q]-\tilde{\mathbf{q}} \tilde{\mathcal{M}}_{0} D^{-1} \tilde{\mathbf{r}}^{\top}
$$

where matrices $J$ and $\tilde{J}$ commute. According to Proposition 2.3 the commutator equation $\left[L_{1}, M_{1}\right]=0$ is equivalent to the system:

$$
\begin{aligned}
& \beta\left[\tilde{J}, Q_{\tau}\right]-\alpha\left[J, Q_{t}\right]+J Q_{x} \tilde{J}-\tilde{J} Q_{x} J+[[J, Q],[\tilde{J}, Q]]+\left[J, \tilde{\mathbf{q}} \tilde{\mathcal{M}}_{0} \tilde{\mathbf{r}}^{\top}\right]+\left[\mathbf{q} \mathcal{M}_{0} \mathbf{r}^{\top}, \tilde{J}\right]=0, \\
& \beta \tilde{\mathbf{q}}_{\tau}-J \tilde{\mathbf{q}}_{x}+[J, Q] \tilde{\mathbf{q}}-\mathbf{q} \mathcal{M}_{0} S_{1}=0, \quad-\beta \tilde{\mathbf{r}}_{\tau}^{\top}+\tilde{\mathbf{r}}_{x}^{\top} J+\tilde{\mathbf{r}}^{\top}[J, Q]+S_{2} \mathcal{M}_{0} \mathbf{r}^{\top}=0, \\
& \alpha \mathbf{q}_{t}-\tilde{J} \mathbf{q}_{x}+[\tilde{J}, Q] \mathbf{q}-\tilde{\mathbf{q}} \tilde{\mathcal{M}}_{0} S_{2}=0, \quad-\alpha \mathbf{r}_{t}^{\top}+\mathbf{r}_{x}^{\top} \tilde{J}+\mathbf{r}^{\top}[\tilde{J}, Q]+S_{1} \tilde{\mathcal{M}}_{0} \tilde{\mathbf{r}}^{\top}=0,
\end{aligned}
$$




$$
S_{1, x}=\mathbf{r}^{\top} \tilde{\mathbf{q}}, \quad S_{2, x}=\tilde{\mathbf{r}}^{\top} \mathbf{q} .
$$

The latter system is a generalization of the $N$-wave problem $[2,76,77,84]$. In case we set $Q=0$ we obtain a noncommutative generalization of the nonlinear system of four waves [38, 39]. Under the Hermitian conjugation reduction $\tilde{\mathbf{r}}=\overline{\tilde{\mathbf{q}}}, \mathcal{M}_{0}=\mathcal{M}_{0}^{*}, \tilde{\mathcal{M}}_{0}=\tilde{\mathcal{M}}_{0}^{*}, \mathbf{r}=\overline{\mathbf{q}}, Q=-Q^{*}, \alpha, \beta \in \mathbb{R}$, $J=J^{*}, \tilde{J}=\tilde{J}^{*}$ the latter system reads:

$$
\begin{aligned}
& \beta\left[\tilde{J}, Q_{\tau}\right]-\alpha\left[J, Q_{t}\right]+J Q_{x} \tilde{J}-\tilde{J} Q_{x} J+[[J, Q],[\tilde{J}, Q]] \\
& \quad+\left[J, \tilde{\mathbf{q}} \tilde{\mathcal{M}}_{0} \tilde{\mathbf{q}}^{*}\right]-\left[\tilde{\mathbf{q}} \tilde{\mathcal{M}}_{0} \tilde{\mathbf{q}}^{*}, \tilde{J}\right]=0, \quad S_{1, x}=\mathbf{q}^{*} \tilde{\mathbf{q}}, \\
& \beta \tilde{\mathbf{q}}_{\tau}-J \tilde{\mathbf{q}}_{x}+[J, Q] \tilde{\mathbf{q}}-\mathbf{q} \mathcal{M}_{0} S_{1}=0, \quad \alpha \mathbf{q}_{t}-\tilde{J} \mathbf{q}_{x}+[\tilde{J}, Q] \mathbf{q}-\tilde{\mathbf{q}} \tilde{\mathcal{M}}_{0} S_{1}^{*}=0 .
\end{aligned}
$$

2. $k=1, n=2$.

$$
L_{1}=\beta_{1} \partial_{\tau_{1}}-\mathbf{q} \mathcal{M}_{0} D^{-1} \mathbf{r}^{\top}, \quad M_{2}=\alpha_{2} \partial_{t_{2}}-c D^{2}+v-\tilde{\mathbf{q}} \tilde{\mathcal{M}}_{0} D^{-1} \tilde{\mathbf{r}}^{\top}, \quad c \in \mathbb{C} .
$$

Lax equation $\left[L_{1}, M_{2}\right]=0$ is equivalent to the following generalization of the DS-III equation:

$$
\begin{aligned}
& \beta_{1} \tilde{\mathbf{q}}_{\tau_{1}}=\mathbf{q} \mathcal{M}_{0} S_{1}, \quad \beta_{1} \tilde{\mathbf{r}}_{\tau_{1}}^{\top}=S_{2} \mathcal{M}_{0} \mathbf{r}^{\top}, \quad S_{1, x}=\mathbf{r}^{\top} \tilde{\mathbf{q}}, \\
& \alpha_{2} \mathbf{q}_{t_{2}}-c \mathbf{q}_{x x}+v \mathbf{q}=\tilde{\mathbf{q}} \tilde{\mathcal{M}}_{0} S_{2}, \quad \alpha_{2} \mathbf{r}_{t_{2}}^{\top}+c \mathbf{r}_{x x}^{\top}-\mathbf{r}^{\top} v=S_{1} \tilde{\mathcal{M}}_{0} \tilde{\mathbf{r}}^{\top}, \quad S_{2, x}=\tilde{\mathbf{r}}^{\top} \mathbf{q}, \\
& \beta_{1} v_{\tau_{1}}=2\left(\mathbf{q} \mathcal{M}_{0} \mathbf{r}^{\top}\right)_{x} .
\end{aligned}
$$

If we set $\tilde{\mathbf{q}}=0, \tilde{\mathbf{r}}=0$ we recover the system

$$
\alpha_{2} \mathbf{q}_{t_{2}}-c \mathbf{q}_{x x}+v \mathbf{q}=0, \quad \alpha_{2} \mathbf{r}_{t_{2}}^{\top}+c \mathbf{r}_{x x}^{\top}-\mathbf{r}^{\top} v=0, \quad \beta_{1} v_{\tau_{1}}=2\left(\mathbf{q} \mathcal{M}_{0} \mathbf{r}^{\top}\right)_{x},
$$

which under reduction $\alpha_{2} \in \mathrm{i} \mathbb{R}, \beta_{1}, c \in \mathbb{R}, \mathcal{M}_{0}=\mathcal{M}_{0}^{*}, v=v^{*}, \mathbf{r}=\overline{\mathbf{q}}$ becomes the matrix version of the DS-III system (see [26]):

$$
\alpha_{2} \mathbf{q}_{t_{2}}-c \mathbf{q}_{x x}+v \mathbf{q}=0, \quad \beta_{1} v_{\tau_{1}}=2\left(\mathbf{q} \mathcal{M}_{0} \mathbf{q}^{*}\right)_{x} .
$$

3. $k=3, n=2$. In this case we obtain the following pair of operators:

$$
\begin{aligned}
& L_{3}=\beta_{3} \partial_{\tau_{3}}-c_{1}\left(D^{3}-w D-u\right)-\mathbf{q} \mathcal{M}_{0} D^{-1} \mathbf{r}^{\top}, \\
& M_{2}=\alpha_{2} \partial_{t_{2}}-c_{2}\left(D^{2}-v\right)-\tilde{\mathbf{q}} \tilde{\mathcal{M}}_{0} D^{-1} \tilde{\mathbf{r}}^{\top}
\end{aligned}
$$

Equation $\left[L_{3}, M_{2}\right]=0$ is equivalent to the following system:

$$
\begin{aligned}
& c_{1} c_{2}(2 w-3 v)=0, \quad-\alpha_{2} c_{1} w_{t_{2}}-\frac{3}{2} c_{1} c_{2} v_{x x}+3 c_{1}\left(\tilde{\mathbf{q}} \tilde{\mathcal{M}}_{0} \tilde{\mathbf{r}}^{\top}\right)_{x}+2 c_{1} c_{2} u_{x}=0, \\
& \beta_{3} c_{2} v_{\tau_{3}}-c_{1} c_{2} v_{x x x}+3 c_{1}\left(\tilde{\mathbf{q}}_{x} \tilde{\mathcal{M}}_{0} \tilde{\mathbf{r}}^{\top}\right)_{x}+c_{1} c_{2} w v_{x}-c_{1}\left[w, \tilde{\mathbf{q}} \tilde{\mathcal{M}}_{0} \tilde{\mathbf{r}}^{\top}\right] \\
& \quad+c_{1} c_{2}[u, v]+c_{1} c_{2} u_{x x}-\alpha_{2} c_{1} u_{t_{2}}-2 c_{2}\left(\mathbf{q} \mathcal{M}_{0} \mathbf{r}^{\top}\right)_{x}=0, \\
& \beta_{3} \tilde{\mathbf{q}}_{\tau_{3}}-c_{1} \tilde{\mathbf{q}}_{x x x}+c_{1} w \tilde{\mathbf{q}}_{x}+c_{1} u \tilde{\mathbf{q}}-\mathbf{q} \mathcal{M}_{0} S_{1}=0, \quad S_{1, x}=\mathbf{r}^{\top} \tilde{\mathbf{q}}, \\
& \quad-\beta_{3} \tilde{\mathbf{r}}_{\tau_{3}}^{\top}+c_{1} \tilde{\mathbf{r}}_{x x x}^{\top}-c_{1}\left(\tilde{\mathbf{r}}^{\top} w\right)_{x}+c_{1} \tilde{\mathbf{r}}^{\top} u+S_{2} \mathcal{M}_{0} \mathbf{r}^{\top}=0, \quad S_{2, x}=\tilde{\mathbf{r}}^{\top} \mathbf{q}, \\
& \alpha_{2} \mathbf{q}_{t_{2}}-c_{2} \mathbf{q}_{x x}+c_{2} v \mathbf{q}-\tilde{\mathbf{q}} \tilde{\mathcal{M}}_{0} S_{2}=0, \\
& \alpha_{2} \mathbf{r}_{t_{2}}^{\top}+c_{2} \mathbf{r}_{x x}^{\top}-c_{2} \mathbf{r}^{\top} v-S_{1} \tilde{\mathcal{M}}_{0} \tilde{\mathbf{r}}^{\top}=0 .
\end{aligned}
$$

The latter consists of several special cases:

a) $c_{1}=c_{2}=1$. In this case the latter system can be rewritten in the following way:

$$
-\frac{3}{2} \alpha_{2} v_{t_{2}}-\frac{3}{2} v_{x x}+3\left(\tilde{\mathbf{q}} \tilde{\mathcal{M}}_{0} \tilde{\mathbf{r}}^{\top}\right)_{x}+2 u_{x}=0
$$




$$
\begin{aligned}
& \left(\beta_{3} v_{\tau_{3}}-\frac{1}{4} v_{x x x}+\frac{3}{2} v v_{x}\right)_{x}-3 \alpha^{2} v_{t_{2} t_{2}}+\left([u, v]-\left[w, \tilde{\mathbf{q}} \tilde{\mathcal{M}}_{0} \tilde{\mathbf{r}}^{\top}\right]\right)_{x} \\
& \quad+\frac{3}{2}\left(\tilde{\mathbf{q}}_{x x} \tilde{\mathcal{M}}_{0} \tilde{\mathbf{r}}^{\top}-\tilde{\mathbf{q}} \tilde{\mathcal{M}}_{0} \tilde{\mathbf{r}}_{x x}^{\top}+\alpha\left(\tilde{\mathbf{q}} \tilde{\mathcal{M}}_{0} \tilde{\mathbf{r}}^{\top}\right)_{t_{2}}\right)_{x}-2\left(\mathbf{q} \mathcal{M}_{0} \mathbf{r}^{\top}\right)_{x x}=0, \\
& \beta_{3} \tilde{\mathbf{q}}_{\tau_{3}}-\tilde{\mathbf{q}}_{x x x}+\frac{3}{2} v \tilde{\mathbf{q}}_{x}+u \tilde{\mathbf{q}}-\mathbf{q} \mathcal{M}_{0} S_{1}=0, \quad S_{1, x}=\mathbf{r}^{\top} \tilde{\mathbf{q}}, \\
& -\beta_{3} \tilde{\mathbf{r}}_{\tau_{3}}^{\top}+\tilde{\mathbf{r}}_{x x x}^{\top}-\frac{3}{2}\left(\tilde{\mathbf{r}}^{\top} v\right)_{x}+\tilde{\mathbf{r}}^{\top} u+S_{2} \mathcal{M}_{0} \mathbf{r}^{\top}=0, \quad S_{2, x}=\tilde{\mathbf{r}}^{\top} \mathbf{q}, \\
& \alpha_{2} \mathbf{q}_{t_{2}}-\mathbf{q}_{x x}+v \mathbf{q}-\tilde{\mathbf{q}} \tilde{\mathcal{M}}_{0} S_{2}=0, \quad \alpha_{2} \mathbf{r}_{t_{2}}^{\top}+\mathbf{r}_{x x}^{\top}-\mathbf{r}^{\top} v-S_{1} \tilde{\mathcal{M}}_{0} \tilde{\mathbf{r}}^{\top}=0 .
\end{aligned}
$$

In the scalar case $(N=1)$ under the Hermitian conjugation reduction: $\alpha_{2} \in i \mathbb{R}, \mathbf{r}=\overline{\mathbf{q}}$, $\mathcal{M}_{0}=\mathcal{M}_{0}^{*}\left(M_{2}=M_{2}^{*}\right)$ and $\beta_{3} \in \mathbb{R}, \tilde{\mathcal{M}}_{0}=-\tilde{\mathcal{M}}_{0}^{*}, \tilde{\mathbf{r}}=\overline{\tilde{\mathbf{q}}}, w=w^{*}, w_{x}^{*}=u+u^{*}, v=v^{*}$ $\left(L_{3}=-L_{3}^{*}\right)$. the latter equation reads:

$$
\begin{aligned}
& \left(\beta_{3} v_{\tau_{3}}-\frac{1}{4} v_{x x x}+\frac{3}{2} v v_{x}\right)_{x}-3 \alpha^{2} v_{t_{2} t_{2}} \\
& \quad+\frac{3}{2}\left(\tilde{\mathbf{q}}_{x x} \tilde{\mathcal{M}}_{0} \tilde{\mathbf{q}}^{*}-\tilde{\mathbf{q}} \tilde{\mathcal{M}}_{0} \tilde{\mathbf{q}}_{x x}^{*}+\alpha\left(\tilde{\mathbf{q}} \tilde{\mathcal{M}}_{0} \tilde{\mathbf{q}}^{*}\right)_{t}\right)_{x}-2\left(\mathbf{q} \mathcal{M}_{0} \mathbf{q}^{*}\right)_{x x}=0 \\
& \beta_{3} \tilde{\mathbf{q}}_{\tau_{3}}-\tilde{\mathbf{q}}_{x x x}+\frac{3}{2} v \tilde{\mathbf{q}}_{x}+u \tilde{\mathbf{q}}-\mathbf{q} \mathcal{M}_{0} S_{1}=0, \quad S_{1, x}=\mathbf{q}^{*} \tilde{\mathbf{q}} \\
& \alpha_{2} \mathbf{q}_{t_{2}}-\mathbf{q}_{x x}+v \mathbf{q}-\tilde{\mathbf{q}} \tilde{\mathcal{M}}_{0} S_{1}^{*}=0 .
\end{aligned}
$$

This system is a generalization of the KP equation with self-consistent sources (KPSCS). In particular, if we set $\tilde{\mathcal{M}}_{0}=0, \tilde{\mathbf{q}}=0$ we recover KPSCS of the first type

$$
\left(\beta_{3} v_{\tau_{3}}-\frac{1}{4} v_{x x x}+\frac{3}{2} v v_{x}\right)_{x}-3 \alpha^{2} v_{t_{2} t_{2}}=2\left(\mathbf{q} \mathcal{M}_{0} \mathbf{q}^{*}\right)_{x x}, \quad \alpha_{2} \mathbf{q}_{t_{2}}-\mathbf{q}_{x x}+v \mathbf{q}=0 .
$$

In case $\mathcal{M}_{0}=0, \mathbf{q}=0$ in (2.10) we obtain KPSCS of the second type

$$
\begin{aligned}
& \left(\beta_{3} v_{\tau_{3}}-\frac{1}{4} v_{x x x}+\frac{3}{2} v v_{x}\right)_{x}-3 \alpha^{2} v_{t_{2} t_{2}}=-\frac{3}{2}\left(\tilde{\mathbf{q}}_{x x} \tilde{\mathcal{M}}_{0} \tilde{\mathbf{q}}^{*}-\tilde{\mathbf{q}} \tilde{\mathcal{M}}_{0} \tilde{\mathbf{q}}_{x x}^{*}+\alpha\left(\tilde{\mathbf{q}} \tilde{\mathcal{M}}_{0} \tilde{\mathbf{q}}^{*}\right)_{t}\right)_{x}, \\
& \beta_{3} \tilde{\mathbf{q}}_{\tau_{3}}-\tilde{\mathbf{q}}_{x x x}+\frac{3}{2} v \tilde{\mathbf{q}}_{x}+u \tilde{\mathbf{q}}=0 .
\end{aligned}
$$

KPSCS and the respective matrix $(1+1)$-dimensional counterpart (KdV equation with selfconsistent sources) have been investigated recently via Darboux transformations [45, 83] and the inverse scattering method [6].

b) $c_{1}=0, c_{2}=1$. In this case $(2.9)$ becomes the following:

$$
\begin{aligned}
& \beta_{3} v_{\tau_{3}}=2\left(\mathbf{q} \mathcal{M}_{0} \mathbf{r}^{\top}\right)_{x}, \quad \beta_{3} \tilde{\mathbf{q}}_{\tau_{3}}-\mathbf{q} \mathcal{M}_{0} S_{1}=0, \quad-\beta_{3} \tilde{\mathbf{r}}_{\tau_{3}}^{\top}+S_{2} \mathcal{M}_{0} \mathbf{r}^{\top}=0, \\
& \alpha_{2} \mathbf{q}_{t_{2}}-\mathbf{q}_{x x}+v \mathbf{q}-\tilde{\mathbf{q}} \tilde{\mathcal{M}}_{0} S_{1}=0, \quad S_{1, x}=\mathbf{r}^{\top} \tilde{\mathbf{q}}, \\
& \alpha_{2} \mathbf{r}_{t_{2}}^{\top}+\mathbf{r}_{x x}^{\top}-\mathbf{r}^{\top} v-S_{2} \tilde{\mathcal{M}}_{0} \tilde{\mathbf{r}}^{\top}=0, \quad S_{2}=\tilde{\mathbf{r}}^{\top} \mathbf{q} .
\end{aligned}
$$

In case $\alpha_{2} \in \mathrm{i} \mathbb{R}, \beta_{3} \in \mathbb{R}, \mathcal{M}_{0}=\mathcal{M}_{0}^{*}, \mathbf{q}=\overline{\mathbf{r}}, \tilde{\mathcal{M}}_{0}=0, \tilde{\mathbf{q}}=\tilde{\mathbf{r}}=0$ the latter becomes the noncommutative generalization of the DS-III system.

Now we will show that $(2+1)$-BD $k$-cKP hierarchy presented in [11] can be recovered from Lax operators (2.2). At first, let us put in formulae (2.2):

$$
\begin{aligned}
& \tilde{\mathbf{q}}:=\left(\tilde{\mathbf{q}}_{1}, c_{l} \mathbf{q}[0], c_{l} \mathbf{q}[1], \ldots, c_{l} \mathbf{q}[l]\right), \quad \tilde{\mathbf{r}}:=\left(\tilde{\mathbf{r}}_{1}, \mathbf{r}[l], \mathbf{r}[l-1], \ldots, \mathbf{r}[0]\right), \\
& \tilde{\mathcal{M}}_{0}=\operatorname{diag}\left(\tilde{\mathcal{M}}_{1}, I_{l+1} \otimes \mathcal{M}_{0}\right),
\end{aligned}
$$


where $\mathbf{q}[j]=\left(L_{k}\right)^{j}\{\mathbf{q}\}, \mathbf{r}[j]=\left(L_{k}^{\tau}\right)^{j}\{\mathbf{r}\}, j=\overline{0, l}$. I.e., $\tilde{m}=\tilde{m}_{1}+m(l+1)$ and matrices $\tilde{\mathbf{q}}$ and $\tilde{\mathbf{r}}$ consist of $N \times \tilde{m}_{1}$-matrix-valued blocks $\tilde{\mathbf{q}}_{1}$ and $\tilde{\mathbf{r}}_{1}$ and $(N \times m)$-matrix-valued blocks $\mathbf{q}[j]$ and $\mathbf{r}[j], j=\overline{0, l} . \tilde{\mathcal{M}}_{0}$ is a block-diagonal matrix and $I_{l+1} \otimes \mathcal{M}_{0}$ stands for the tensor product of the $(l+1)$-dimensional identity matrix $I_{l+1}$ and matrix $\mathcal{M}_{0}$. Then we get the following operators in $(2.2)$ :

$$
\begin{aligned}
& L_{k}=\beta_{k} \partial_{\tau_{k}}-B_{k}-\mathbf{q} \mathcal{M}_{0} D^{-1} \mathbf{r}^{\top}, \quad B_{k}=\sum_{j=0}^{k} u_{j} D^{j}, u_{j}=u_{j}\left(x, \tau_{k}, t_{n}\right), \quad \beta_{k} \in \mathbb{C}, \\
& M_{n}=M_{n, l}=\alpha_{n} \partial_{t_{n}}-A_{n}-\tilde{\mathbf{q}}_{1} \tilde{\mathcal{M}}_{1} D^{-1} \tilde{\mathbf{r}}_{1}^{\top}-c_{l} \sum_{j=0}^{l} \mathbf{q}[j] \mathcal{M}_{0} D^{-1} \mathbf{r}^{\top}[l-j], \quad l=1, \ldots \\
& A_{n}=\sum_{i=0}^{n} v_{i} D^{i}, v_{i}=v_{i}\left(x, \tau_{k}, t_{n}\right), \quad \alpha_{n} \in \mathbb{C} .
\end{aligned}
$$

The following proposition holds:

Proposition 2.4. Assume that equations

$$
\begin{aligned}
& M_{n, l}\{\mathbf{q}\}=c_{l}\left(L_{k}\right)^{l+1}\{\mathbf{q}\}, \quad M_{n, l}^{\tau}\{\mathbf{r}\}=c_{l}\left(L_{k}^{\tau}\right)^{l+1}\{\mathbf{r}\}, \\
& L_{k}\left\{\tilde{\mathbf{q}}_{1}\right\}=\tilde{\mathbf{q}}_{1} \Lambda_{\tilde{\mathbf{q}}_{1}}, \quad L_{k}^{\tau}\left\{\tilde{\mathbf{r}}_{1}\right\}=\tilde{\mathbf{r}}_{1} \Lambda_{\tilde{\mathbf{r}}_{1}}
\end{aligned}
$$

with constant matrices $\Lambda_{\tilde{\mathbf{q}}_{1}}$ and $\Lambda_{\tilde{\mathbf{r}}_{1}}$ are satisfied, where the latter solve $\Lambda_{\tilde{\mathbf{q}}_{1}} \tilde{\mathcal{M}}_{1}-\tilde{\mathcal{M}}_{1} \Lambda_{\tilde{\mathbf{r}}_{1}}^{\top}=0$. Then Lax equation $\left[L_{k}, M_{n, l}\right]=0$ holds if and only if its differential part is equal to zero, i.e., $\left[L_{k}, M_{n, l}\right]_{\geq 0}=0$.

Proof. The proof is similar to the proof of the Proposition 2.3 and the proof of the Theorem 1 in [11].

Remark 2.5. Setting $\tilde{\mathbf{q}}_{1}=0$ and $\tilde{\mathbf{r}}_{1}=0$ in $(2.12)$ we recover $(2+1)$-BDk-cKP hierarchy (Lax pairs (2.12) with equations (2.13)) that contains the following subcases:

1. $\beta_{k}=0, c_{l}=0$. Under this assumption we obtain matrix $k$-constrained KP hierarchy [58]. We shall point out that the case $\beta_{k}=0$ and $c_{l} \neq 0$ also leads to matrix $k$-constrained KP hierarchy.

2. $c_{l}=0, N=1, v_{n}=u_{k}=1, v_{n-1}=u_{k-1}=0$. In this way we recover $(2+1)$-dimensional $k$ cKP hierarchy [73].

3. $n=0$. The differential part of $M_{0, l}(2.12)$ is equal to zero in this case $\left(A_{0}=0\right)$ and we get a new generalization of DS-III hierarchy.

4. $c_{l}=0$. We obtain $\left(t_{A}, \tau_{B}\right)$-matrix KP hierarchy that was investigated in [36].

5. If $l=0$ we recover $\left(\gamma_{A}, \sigma_{B}\right)$-matrix KP hierarchy [37].

6. Case $N=1, \mathbf{q}=0, \mathbf{r}=0$ leads to KP hierarchy.

\section{Dressing methods for the new $(2+1)$-dimensional generalizations of $k$-constrained KP hierarchy}

\subsection{Dressing via Darboux transformations}

In this section we will consider Darboux transformations (DT) for the pair of operators (2.2) and its reduction (2.12). At first, we shall start with the linear problem associated with the 
operator $L_{k}(2.2)$ :

$$
L_{k}\left\{\varphi_{1}\right\}=\beta_{k}\left(\varphi_{1}\right)_{\tau_{k}}-\sum_{j=0}^{k} u_{j}\left(\varphi_{1}\right)^{(j)}-\mathbf{q} \mathcal{M}_{0} D^{-1}\left\{\mathbf{r}^{\top} \varphi_{1}\right\}=\varphi_{1} \Lambda_{1},
$$

where $\varphi_{1}$ is $(N \times N)$-matrix-valued function; $\Lambda_{1}$ is a constant matrix with dimension $N \times N$. Introduce the DT in the following way:

$$
W_{1}\left[\varphi_{1}\right]=\varphi_{1} D \varphi_{1}^{-1}=D-\varphi_{1, x} \varphi_{1}^{-1} .
$$

The following proposition holds.

Proposition 3.1. The operator $\hat{L}_{k}[1]:=W_{1}\left[\varphi_{1}\right] L_{k} W_{1}^{-1}\left[\varphi_{1}\right]$ obtained from $L_{k}(2.2)$ via DT (3.1) has the form

$$
\hat{L}_{k}[1]:=W_{1}\left[\varphi_{1}\right] L_{k} W_{1}^{-1}\left[\varphi_{1}\right]=\beta_{k} \partial_{\tau_{k}}-\hat{B}_{k}-\hat{\mathbf{q}}_{1} \mathcal{M}_{0} D^{-1} \hat{\mathbf{r}}_{1}^{\top}, \quad \hat{B}_{k}[1]=\sum_{j=0}^{k} \hat{u}_{j}[1] D^{j},
$$

where

$$
\hat{\mathbf{q}}_{1}=W_{1}\left[\varphi_{1}\right]\{\mathbf{q}\}, \quad \hat{\mathbf{r}}_{1}=W_{1}^{-1, \tau}\left[\varphi_{1}\right]\{\mathbf{r}\} .
$$

$\hat{u}_{j}[1]$ are $(N \times N)$-matrix coefficients depending on function $\varphi_{1}$ and coefficients $u_{i}, i=\overline{0, k}$. In particular, $\hat{u}_{k}[1]=u_{k}$.

Proof. It is evident that the inverse operator to (3.1) has the form $W_{1}^{-1}\left[\varphi_{1}\right]=\varphi_{1} D^{-1} \varphi_{1}^{-1}$. Thus, we have

$$
\begin{aligned}
\hat{L}_{k}[1] & =W_{1}\left[\varphi_{1}\right] L_{k} W_{1}^{-1}\left[\varphi_{1}\right]=\varphi_{1} D \varphi_{1}^{-1}\left(\beta_{k} \partial_{\tau_{k}}-B_{k}-\mathbf{q} \mathcal{M}_{0} D^{-1} \mathbf{r}^{\top}\right) \varphi_{1} D^{-1} \varphi_{1}^{-1} \\
& =\beta_{k} \partial_{\tau_{k}}+\left(\hat{L}_{k}[1]\right)_{\geq 0}+\left(\hat{L}_{k}[1]\right)_{<0}
\end{aligned}
$$

where $\left(\hat{L}_{k}[1]\right)_{\geq 0}=-\hat{B}_{k}[1]=-\sum_{j=0}^{k} \hat{u}_{j}[1] D^{j}$. It remains to find the explicit form of $\left(\hat{L}_{k}\right)_{<0}$. Using formulae (2.3)-(2.5) we have:

$$
\begin{aligned}
\left(\hat{L}_{k}[1]\right)_{<0}= & \left(\beta_{k} \varphi_{1} D \varphi_{1}^{-1} \varphi_{1, \tau_{k}} D^{-1} \varphi_{1}^{-1}\right)_{<0}-\left(\varphi_{1} D \varphi_{1}^{-1} B_{k}\left\{\varphi_{1}\right\} D^{-1} \varphi_{1}^{-1}\right)_{<0} \\
& -\left(\varphi_{1} D \varphi_{1}^{-1} \mathbf{q} \mathcal{M}_{0} D^{-1}\left\{\mathbf{r}^{\top} \varphi_{1}\right\} D^{-1} \varphi_{1}^{-1}-\varphi_{1} D \varphi_{1}^{-1} \mathbf{q} \mathcal{M}_{0} D^{-1} D^{-1}\left\{\mathbf{r}^{\top} \varphi_{1}\right\} \varphi_{1}^{-1}\right)_{<0} \\
= & \left(\varphi_{1} D \varphi_{1}^{-1} \varphi_{1} \Lambda_{1} D^{-1} \varphi_{1}^{-1}\right)_{<0}+\varphi_{1} D\left\{\varphi_{1}^{-1} \mathbf{q}\right\} \mathcal{M}_{0} D^{-1} D^{-1}\left\{\mathbf{r}^{\top} \varphi_{1}\right\} \varphi_{1}^{-1} \\
= & -W_{1}\{\mathbf{q}\} \mathcal{M}_{0} D^{-1}\left(W_{1}^{-1, \tau}\{\mathbf{r}\}\right)^{\top} .
\end{aligned}
$$

It is also possible to generalize the latter theorem to the case of finite number of solutions of linear problems associated with the operator $L_{k}$. Namely, let functions $\varphi_{s}, s=\overline{1, K}$ be solutions of the problems:

$$
L_{k}\left\{\varphi_{s}\right\}=\beta_{k}\left(\varphi_{s}\right)_{\tau_{k}}-\sum_{j=0}^{k} u_{j}\left(\varphi_{s}\right)^{(j)}-\mathbf{q} \mathcal{M}_{0} D^{-1}\left\{\mathbf{r}^{\top} \varphi_{s}\right\}=\varphi_{s} \Lambda_{s}, \quad s=\overline{1, K} .
$$

For further convenience we shall use the notations $\varphi_{s}[1]:=\varphi_{s}, s=\overline{1, K}$ and define the following functions:

$$
\varphi_{s}[2]=W_{1}\left[\varphi_{1}[1]\right]\left\{\varphi_{s}[1]\right\}, \quad s=\overline{2, K} .
$$


Now, using functions $\varphi_{1}[1], \varphi_{2}[2]$, we shall define functions $\varphi_{s}[3], s=\overline{3, K}$ :

$$
\varphi_{s}[3]:=W_{1}\left[\varphi_{2}[2]\right]\left\{\varphi_{s}[2]\right\}=W_{1}\left[\varphi_{2}[2]\right] W_{1}\left[\varphi_{1}[1]\right]\left\{\varphi_{s}[1]\right\}, \quad s=\overline{3, K}
$$

At the $p$-th step we obtain functions: $\varphi_{s}[p]:=W_{1}\left[\varphi_{p-1}[p-1]\right]\left\{\varphi_{s}[p-1]\right\}=W_{1}\left[\varphi_{p-1}[p-\right.$ $1]] \cdots W_{1}\left[\varphi_{2}[2]\right] W_{1}\left[\varphi_{1}[1]\right]\left\{\varphi_{s}[1]\right\}, s=\overline{p, K}$. Now we shall construct the following generalization of DT (3.1):

$$
\begin{aligned}
& W_{K}\left[\varphi_{1}, \ldots, \varphi_{K}\right]=W_{1}\left[\varphi_{K}[K]\right] \cdots W_{1}\left[\varphi_{1}[1]\right] \\
& \quad=\left(D-\varphi_{K, x}[K] \varphi_{K}^{-1}[K]\right) \cdots\left(D-\varphi_{1, x}[1] \varphi_{1}^{-1}[1]\right) .
\end{aligned}
$$

The following statement holds:

Proposition 3.2. The operator

$$
\hat{L}_{k}[K]:=W_{K}\left[\varphi_{1}[1], \varphi_{2}[1], \ldots, \varphi_{K}[1]\right] L_{k} W_{K}^{-1}\left[\varphi_{1}[1], \varphi_{2}[1], \ldots, \varphi_{K}[1]\right]=W_{K} L_{k} W_{K}^{-1}
$$

obtained from $L_{k}(2.12)$ via DT (3.4) has the form

$$
\hat{L}_{k}[K]:=W_{K} L_{k} W_{K}^{-1}=\beta_{k} \partial_{\tau_{k}}-\hat{B}_{k}[K]-\hat{\mathbf{q}}_{K} \mathcal{M}_{0} D^{-1} \hat{\mathbf{r}}_{K}^{\top}, \quad \hat{B}_{k}[K]=\sum_{j=0}^{k} \hat{u}_{j}[K] D^{j},
$$

where

$$
\hat{\mathbf{q}}_{K}=W_{K}\left[\varphi_{1}[1], \ldots, \varphi_{K}[1]\right]\{\mathbf{q}\}, \quad \hat{\mathbf{r}}_{K}=W_{K}^{-1, \tau}\left[\varphi_{1}[1], \ldots, \varphi_{K}[1]\right]\{\mathbf{r}\} .
$$

$\hat{u}_{j}[K]$ are $(N \times N)$-matrix coefficients depending on functions $\varphi_{s}, s=\overline{1, K}$ and coefficients $u_{i}$, $i=\overline{0, k}$. In particular, $\hat{u}_{k}[K]=u_{k}$.

Proof. The proof can be done via induction by $K$. Namely, assume that the statement holds for $K-1$. I.e.,

$$
\hat{L}_{k}[K-1]=W_{K-1} L_{k} W_{K-1}^{-1}=\beta_{k} \partial_{\tau_{k}}-\hat{B}_{k}[K-1]-\hat{\mathbf{q}}_{K-1} \mathcal{M}_{0} D^{-1} \hat{\mathbf{r}}_{K-1}^{\top},
$$

with $\hat{\mathbf{q}}_{K-1}=W_{K-1}\left[\varphi_{1}[1], \ldots, \varphi_{K-1}[1]\right]\{\mathbf{q}\}$ and $\hat{\mathbf{r}}_{K-1}=W_{K-1}^{-1, \tau}\left[\varphi_{1}[1], \ldots, \varphi_{K-1}[1]\right]\{\mathbf{r}\}$. The function $\varphi_{K}[K]=W_{K-1}\left\{\varphi_{K}[1]\right\}=W_{K-1}\left[\varphi_{1}, \ldots, \varphi_{K-1}\right]\left\{\varphi_{K}[1]\right\}$ (see formulae (3.3), (3.4)) satisfies the equation: $\hat{L}_{k}[K-1]\left\{\varphi_{K}[K]\right\}=W_{K-1} L_{k} W_{K-1}^{-1}\left\{W_{K-1}\left\{\varphi_{K}[1]\right\}\right\}=$ $=W_{K-1} L_{k}\left\{\varphi_{K}[1]\right\}=\varphi_{K}[K] \Lambda_{K}$.

Now, it remains to apply Proposition 3.1 to operator $\hat{L}_{k}[K-1](3.5)$ with the DT $W_{1}\left[\varphi_{K}[K]\right]$ (see formula (3.1)) and use formula $W_{K}=W_{1}\left[\varphi_{K}[K]\right] W_{K-1}$ that immediately follows from (3.4).

Remark 3.3. We shall also point out that in a scalar case $(N=1)$ the DT $W_{K}(3.4)$ can be rewritten in the following way:

$$
W_{K}:=\frac{1}{\mathcal{W}\left[\varphi_{1}, \varphi_{2}, \ldots, \varphi_{K}\right]}\left|\begin{array}{cccc}
\varphi_{1} & \ldots & \varphi_{K} & 1 \\
\varphi_{1}^{\prime} & \ldots & \varphi_{K}^{\prime} & D \\
\ldots & \ldots & \ldots & \ldots \\
\varphi_{1}^{(K)} & \ldots & \varphi_{K}^{(K)} & D^{K}
\end{array}\right|=D^{K}+\sum_{i=0}^{K-1} w_{i} D^{i},
$$

where $\mathcal{W}\left[\varphi_{1}, \varphi_{2}, \ldots, \varphi_{K}\right]$ denotes the Wronskian constructed by solutions $\varphi_{j}, j=1, \ldots, K$, of the linear problem (3.2). It acts on the vector-valued function $\mathbf{q}=\left(q_{1}, \ldots, q_{m}\right)$ in the following way: $W_{K}\{\mathbf{q}\}=\left(W_{K}\left\{q_{1}\right\}, \ldots, W_{K}\left\{q_{m}\right\}\right)$, where $W_{K}\left\{q_{j}\right\}=\frac{\mathcal{W}\left[\varphi_{1}, \varphi_{2}, \ldots, \varphi_{K}, q_{j}\right]}{\mathcal{W}\left[\varphi_{1}, \varphi_{2}, \ldots, \varphi_{K}\right]}$. 
The Darboux transformations (3.1), (3.4) are widely used for the solution generating technique involving Lax pairs consisting of differential operators [29, 30, 50, 51, 66]. Corresponding extensions to integro-differential cases of Lax pairs were made in $[47,54,58,60,73]$ to construct solutions of constrained KP hierarchies and their generalizations. Transformations that generalize (3.1), (3.4) also arise in the bidifferential calculus approach to integrable systems and their hierarchies $[16,18]$. In contrast to $(3.4)$, formula (3.6) does not require iterative applications of DTs and therefore can be used more effectively in the scalar case. We point out that in the matrix (noncommutative) case (3.6) is not valid anymore. However, the corresponding quasideterminant representations can be used (see, e.g., [29, 30]).

From Proposition 3.2 we obtain the corollary for Lax pairs (2.2). Namely, let functions $\varphi_{s}$, $s=\overline{1, K}$ be solutions of the problems:

$$
\begin{aligned}
& L_{k}\left\{\varphi_{s}\right\}=\beta_{k}\left(\varphi_{s}\right)_{\tau_{k}}-\sum_{j=0}^{k} u_{j}\left(\varphi_{s}\right)^{(j)}-\mathbf{q} \mathcal{M}_{0} D^{-1}\left\{\mathbf{r}^{\top} \varphi_{s}\right\}=\varphi_{s} \Lambda_{s}, \\
& M_{n}\left\{\varphi_{s}\right\}=\alpha_{n}\left(\varphi_{s}\right)_{t_{n}}-\sum_{i=0}^{n} v_{i}\left(\varphi_{s}\right)^{(i)}-\tilde{\mathbf{q}} \tilde{\mathcal{M}}_{0} D^{-1}\left\{\tilde{\mathbf{r}}^{\top} \varphi_{s}\right\}=\varphi_{s} \tilde{\Lambda}_{s}, \quad s=\overline{1, K} .
\end{aligned}
$$

Then the following statement holds:

Corollary 3.4. Assume that Lax equation with operators $L_{k}$ and $M_{n}(2.2)$ holds: $\left[L_{k}, M_{n}\right]=0$. Then:

1. Transformed operators

$$
\begin{aligned}
& \hat{L}_{k}[K]:=W_{K}\left[\varphi_{1}, \ldots, \varphi_{K}\right] L_{k} W_{K}^{-1}\left[\varphi_{1}, \ldots, \varphi_{K}\right], \\
& \hat{M}_{n}[K]:=W_{K}\left[\varphi_{1}, \ldots, \varphi_{K}\right] M_{n} W_{K}^{-1}\left[\varphi_{1}, \ldots, \varphi_{K}\right],
\end{aligned}
$$

where $W_{K}$ is defined by (3.4), have the form:

$$
\begin{aligned}
& \hat{L}_{k}[K]:=W_{K} L_{k} W_{K}^{-1}=\beta_{k} \partial_{\tau_{k}}-\hat{B}_{k}[K]-\hat{\mathbf{q}}_{K} \mathcal{M}_{0} D^{-1} \hat{\mathbf{r}}_{K}^{\top}, \\
& \hat{M}_{n}[K]:=W_{K} M_{n} W_{K}^{-1}=\alpha_{n} \partial_{t_{n}}-\hat{A}_{n}[K]-\hat{\tilde{\mathbf{q}}}_{K} \tilde{\mathcal{M}}_{0} D^{-1} \hat{\tilde{\mathbf{r}}}_{K}^{\top}, \\
& \hat{A}_{n}[K]=\sum_{i=0}^{n} \hat{v}_{i}[K] D^{i}, \quad \hat{B}_{k}[K]=\sum_{j=0}^{k} \hat{u}_{j}[K] D^{j},
\end{aligned}
$$

where

$$
\begin{array}{lll}
\hat{\mathbf{q}}_{K}=W_{K}\left[\varphi_{1}[1], \ldots, \varphi_{K}[1]\right]\{\mathbf{q}\}, & \hat{\mathbf{r}}_{K}=W_{K}^{-1, \tau}\left[\varphi_{1}[1], \ldots, \varphi_{K}[1]\right]\{\mathbf{r}\}, \\
\hat{\tilde{\mathbf{q}}}_{K}=W_{K}\left[\varphi_{1}[1], \ldots, \varphi_{K}[1]\right]\{\tilde{\mathbf{q}}\}, & \hat{\tilde{\mathbf{r}}}_{K}=W_{K}^{-1, \tau}\left[\varphi_{1}[1], \ldots, \varphi_{K}[1]\right]\{\tilde{\mathbf{r}}\} .
\end{array}
$$

2. The operators $\hat{L}_{k}[K]$ and $\hat{M}_{n}[K]$ (3.7) satisfy Lax equation: $\left[\hat{L}_{k}[K], \hat{M}_{n}[K]\right]=0$.

3. In case of reduction (2.11) in Lax pair (2.2) we have:

$$
\hat{\tilde{\mathbf{q}}}_{K}=\left(\hat{\tilde{\mathbf{q}}}_{1, K}, c_{l} \hat{\mathbf{q}}_{K}[0], \ldots, c_{l} \hat{\mathbf{q}}_{K}[l]\right), \quad \hat{\tilde{\mathbf{r}}}_{K}=\left(\hat{\tilde{\mathbf{r}}}_{1, K}, \hat{\mathbf{r}}_{K}[0], \ldots \hat{\mathbf{r}}_{K}[l]\right),
$$

where

$$
\begin{aligned}
& \hat{\tilde{\mathbf{q}}}_{1, K}=W_{K}\left\{\tilde{\mathbf{q}}_{1}\right\}, \quad \hat{\tilde{\mathbf{r}}}_{1, K}=W_{K}^{-1, \tau}\left\{\tilde{\mathbf{r}}_{1}\right\}, \\
& \hat{\mathbf{q}}[j]=\left(\hat{L}_{k}[K]\right)^{j}\left\{\hat{\mathbf{q}}_{K}\right\}, \quad \hat{\mathbf{r}}[j]=\left(\hat{L}_{k}^{\tau}[K]\right)^{j}\left\{\hat{\mathbf{r}}_{K}\right\}, \quad j=\overline{0, l} .
\end{aligned}
$$


Proof. 1. Form (3.7) of operators $\hat{L}_{k}[K], \hat{M}_{n}[K]$ follows from Proposition 3.2.

2. We obtain the proof of this item from the following formulae

$$
\left[\hat{L}_{k}[K], \hat{M}_{n}[K]\right]=\left[W_{K} L_{k} W_{K}^{-1}, W_{K} M_{n} W_{K}^{-1}\right]=W_{K}\left[L_{k}, M_{n}\right] W_{K}^{-1}=0 .
$$

3. From formulae:

$$
\begin{aligned}
& \hat{\tilde{\mathbf{q}}}_{K}=W_{K}\left\{\left(\tilde{\mathbf{q}}_{1}, c_{l} \mathbf{q}[0], \ldots, c_{l} \mathbf{q}[l]\right)\right\}=\left(W_{K}\left\{\tilde{\mathbf{q}}_{1}\right\}, c_{l} W_{K}\{\mathbf{q}[0]\}, \ldots, c_{l} W_{K}\{\mathbf{q}[l]\}\right), \\
& W_{K}\{\mathbf{q}[j]\}=W_{K}\left\{\left(L_{k}\right)^{j}\{\mathbf{q}\}\right\}=\left(W_{K} L_{k} W_{K}^{-1}\right)\left\{W_{K}\{\mathbf{q}\}\right\}=\hat{L}_{k}[K]\left\{\hat{\mathbf{q}}_{K}\right\}
\end{aligned}
$$

we get the form of $\hat{\tilde{\mathbf{q}}}_{K}$ mentioned in item 3 . The form of $\hat{\tilde{\mathbf{r}}}_{K}$ can be obtained in a similar way.

\subsection{Dressing via binary Darboux transformations}

In this section we will show that results of paper [80] on binary Darboux transformations (BDT) for linear integro-differential operators can be extended to families of Lax pairs given by (2.2). Namely, let $(N \times K)$-matrix functions $\varphi$ and $\psi$ be solutions of linear problems:

$$
L_{k}\{\varphi\}=\varphi \Lambda_{k}, \quad L_{k}^{\tau}\{\psi\}=\psi \tilde{\Lambda}_{k}, \quad \Lambda_{k}, \tilde{\Lambda}_{k} \in \operatorname{Mat}_{K \times K}(\mathbb{C}) .
$$

Following [80] we introduce BDT in the following way:

$$
W=I-\varphi\left(C+D^{-1}\left\{\psi^{\top} \varphi\right\}\right)^{-1} D^{-1} \psi^{\top},
$$

where $C$ is a $K \times K$-constant nondegenerate matrix. The inverse operator $W^{-1}$ has the form:

$$
W^{-1}=I+\varphi D^{-1}\left(C+D^{-1}\left\{\psi^{\top} \varphi\right\}\right)^{-1} \psi^{\top} .
$$

The following theorem is proven in [80].

Theorem 3.5. The operator $\hat{L}_{k}:=W L_{k} W^{-1}$ obtained from $L_{k}$ in (2.2) via BDT (3.8) has the form

$$
\hat{L}_{k}:=W L_{k} W^{-1}=\beta_{k} \partial_{\tau_{k}}-\hat{B}_{k}-\hat{\mathbf{q}} \mathcal{M}_{0} D^{-1} \hat{\mathbf{r}}^{\top}+\Phi \mathcal{M}_{k} D^{-1} \Psi^{\top}, \quad \hat{B}_{k}=\sum_{j=0}^{k} \hat{u}_{j} D^{j}
$$

where

$$
\begin{aligned}
& \mathcal{M}_{k}=C \Lambda_{k}-\tilde{\Lambda}_{k}^{\top} C, \quad \Phi=\varphi \Delta^{-1}, \quad \Psi=\psi \Delta^{-1, \top}, \quad \Delta=C+D^{-1}\left\{\psi^{\top} \varphi\right\}, \\
& \hat{\mathbf{q}}=W\{\mathbf{q}\}, \quad \hat{\mathbf{r}}=W^{-1, \tau}\{\mathbf{r}\} .
\end{aligned}
$$

$\hat{u}_{j}$ are $(N \times N)$-matrix coefficients depending on functions $\varphi, \psi$ and $u_{j}$. In particular,

$$
\hat{u}_{k}=u_{k}, \quad \hat{u}_{k-1}=u_{k-1}+\left[u_{k}, \varphi\left(C+D^{-1}\left\{\psi^{\top} \varphi\right\}\right)^{-1} \psi^{\top}\right] .
$$

Solution generating method for the hierarchy (2.2)-(2.6) is given by the corollary, which follows from the previous theorem.

Corollary 3.6. Let $(N \times K)$-matrix functions $\varphi$ and $\psi$ satisfy equations:

$$
\begin{aligned}
& L_{k}\{\varphi\}=\varphi \Lambda_{k, 1}, L_{k}^{\tau}\{\psi\}=\psi \tilde{\Lambda}_{k, 1}, \quad \Lambda_{k, 1}, \tilde{\Lambda}_{k, 1} \in \operatorname{Mat}_{K \times K}(\mathbb{C}), \\
& M_{n}\{\varphi\}=\varphi \Lambda_{n, 2}, M_{k}^{\tau}\{\psi\}=\psi \tilde{\Lambda}_{n, 2}, \quad \Lambda_{n, 2}, \tilde{\Lambda}_{n, 2} \in \operatorname{Mat}_{K \times K}(\mathbb{C})
\end{aligned}
$$


with operators $L_{k}$ and $M_{n}(2.2)$ satisfying $\left[L_{k}, M_{n}\right]=0$. Then transformed operators $\hat{L}_{k}$ and $\hat{M}_{n}$ satisfy Lax equation $\left[\hat{L}_{k}, \hat{M}_{n}\right]=0$ and have the form:

$$
\begin{array}{ll}
\hat{L}_{k}:=W L_{k} W^{-1}=\beta_{k} \partial_{\tau_{k}}-\hat{B}_{k}-\hat{\mathbf{q}} \mathcal{M}_{0} D^{-1} \hat{\mathbf{r}}^{\top}+\Phi \mathcal{M}_{k, 1} D^{-1} \Psi^{\top}, & \hat{B}_{k}=\sum_{j=0}^{k} \hat{u}_{j} D^{j}, \\
\hat{M}_{n}:=W M_{n} W^{-1}=\alpha_{n} \partial_{t_{n}}-\hat{A}_{n}-\hat{\tilde{\mathbf{q}}} \tilde{\mathcal{M}}_{0} D^{-1} \hat{\tilde{\mathbf{r}}}^{\top}+\Phi \mathcal{M}_{n, 2} D^{-1} \Psi^{\top}, & \hat{A}_{n}=\sum_{i=0}^{n} \hat{v}_{i} D^{i}
\end{array}
$$

where

$$
\begin{array}{lcl}
\mathcal{M}_{k, 1}=C \Lambda_{k, 1}-\tilde{\Lambda}_{k, 1}^{\top} C, & \mathcal{M}_{n, 2}=C \Lambda_{n, 2}-\tilde{\Lambda}_{n, 2}^{\top} C, \quad \Phi=\varphi \Delta^{-1}, & \Psi=\psi \Delta^{-1, \top}, \\
\Delta=C+D^{-1}\left\{\psi^{\top} \varphi\right\}, & \hat{\mathbf{q}}=W\{\mathbf{q}\}, \quad \hat{\mathbf{r}}=W^{-1, \tau}\{\mathbf{r}\}, \hat{\tilde{\mathbf{q}}}=W\{\tilde{\mathbf{q}}\}, & \hat{\tilde{\mathbf{r}}}=W^{-1, \tau}\{\tilde{\mathbf{r}}\} .
\end{array}
$$

$\hat{u}_{j}$ are $(N \times N)$-matrix coefficients depending on functions $\varphi, \psi$ and $u_{j}, v_{i}$. In particular,

$$
\begin{aligned}
& \hat{u}_{k}=u_{k}, \hat{u}_{k-1}=u_{k-1}+\left[u_{k}, \varphi\left(C+D^{-1}\left\{\psi^{\top} \varphi\right\}\right)^{-1} \psi^{\top}\right], \\
& \hat{v}_{n}=v_{n}, \hat{v}_{n-1}=v_{n-1}+\left[v_{n}, \varphi\left(C+D^{-1}\left\{\psi^{\top} \varphi\right\}\right)^{-1} \psi^{\top}\right] .
\end{aligned}
$$

Proof. From formulae $W\left[L_{k}, M_{n}\right] W^{-1}=\left[\hat{L}_{k}, \hat{M}_{n}\right]=0$ we obtain that Lax equation with transformed operators is satisfied. Form (3.9) of the transformed operators $\hat{L}_{k}$ and $\hat{M}_{n}$ follows from Theorem 3.5.

BDTs were used to generate solutions of the constrained KP hierarchies in $[56,82]$ and they were also applied to $(2+1)$-BD $k$-cKP hierarchy in [11]. Theorem 3.5 with Corollary 3.6 extend the respective results to $(2+1)$-dimensional generalizations of the $k$-constrained $\mathrm{KP}$ hierarchy (2.2), (2.6).

\section{New $(2+1)$-dimensional generalizations of the modified $k$-constrained KP hierarchy}

We will investigate the following (2+1)-dimensional generalizations of the modified $k$-constrained $\mathrm{KP}(k$-cmKP) hierarchy:

$$
\begin{array}{ll}
L_{k}=\beta_{k} \partial_{\tau_{k}}-B_{k}-\mathbf{q} \mathcal{M}_{0} D^{-1} \mathbf{r}^{\top} D, & B_{k}=\sum_{j=1}^{k} u_{j} D^{j}, \quad u_{j}=u_{j}\left(x, \tau_{k}, t_{n}\right), \quad \beta_{k} \in \mathbb{C}, \\
M_{n}=\alpha_{n} \partial_{t_{n}}-A_{n}-\tilde{\mathbf{q}} \tilde{\mathcal{M}}_{0} D^{-1} \tilde{\mathbf{r}}^{\top} D, & A_{n}=\sum_{i=1}^{n} v_{i} D^{i}, \quad v_{i}=v_{i}\left(x, \tau_{k}, t_{n}\right), \quad \alpha_{n} \in \mathbb{C},(
\end{array}
$$

where $u_{j}$ and $v_{i}$ are matrix-valued functions of dimension $N \times N ; \mathbf{q}$ and $\mathbf{r}$ are matrix-valued functions of dimension $N \times m ; \tilde{\mathbf{q}}$ and $\tilde{\mathbf{r}}$ are matrix-valued functions with dimension $N \times \tilde{m} . \mathcal{M}_{0}$ and $\tilde{\mathcal{M}}_{0}$ are constant matrices with dimensions $m \times m$ and $\tilde{m} \times \tilde{m}$ respectively.

Using equality (2.4) we can rewrite operators (4.1) as

$$
\begin{aligned}
& L_{k}=\beta_{k} \partial_{\tau_{k}}-B_{k}-\mathbf{q} \mathcal{M}_{0} \mathbf{r}^{\top}+\mathbf{q} \mathcal{M}_{0} D^{-1} \mathbf{r}_{x}^{\top}, \\
& M_{n}=\alpha_{n} \partial_{t_{n}}-A_{n}-\tilde{\mathbf{q}} \tilde{\mathcal{M}}_{0} \tilde{\mathbf{r}}^{\top}+\tilde{\mathbf{q}} \tilde{\mathcal{M}}_{0} D^{-1} \tilde{\mathbf{r}}_{x}^{\top} .
\end{aligned}
$$

From the latter it becomes clear that (4.1) can be considered as a reduction in (2.2) $\left(B_{k} \rightarrow\right.$ $\left.B_{k}+\mathbf{q} \mathcal{M}_{0} \mathbf{r}^{\top}, \mathbf{r}^{\top} \rightarrow-\mathbf{r}_{x}^{\top}\right)$. However, Lax pairs (4.1) provide us with different (2+1)-dimensional 
equations (see (4.4) and (4.5)). In addition, they require different kind of BDT (see Theorem 4.2 with its corollary) for the corresponding solution generating technique.

Setting $\tilde{\mathbf{q}}=0, \tilde{\mathbf{r}}=0, N=1$ in $(4.1)$ we recover $(2+1)$-dimensional $k$-cmKP hierarchy [47].

The following proposition holds:

Proposition 4.1. Lax equation $\left[L_{k}, M_{n}\right]=0$ is satisfied in case the following equations hold:

$$
\begin{aligned}
& {\left[L_{k}, M_{n}\right]_{\geq 0}=0, \quad L_{k}\{\tilde{\mathbf{q}}\}=\tilde{\mathbf{q}} \Lambda_{\tilde{\mathbf{q}}}, \quad\left(D^{-1} L_{k}^{\tau} D\right)\{\tilde{\mathbf{r}}\}=\tilde{\mathbf{r}} \Lambda_{\tilde{\mathbf{r}}},} \\
& M_{n}\{\mathbf{q}\}=\mathbf{q} \Lambda_{\mathbf{q}}, \quad\left(D^{-1} M_{n}^{\tau} D\right)\{\mathbf{r}\}=\mathbf{r} \Lambda_{\mathbf{r}},
\end{aligned}
$$

where $\Lambda_{\mathbf{q}}, \Lambda_{\mathbf{r}}, \Lambda_{\tilde{\mathbf{q}}}, \Lambda_{\tilde{\mathbf{r}}}$ are constant matrices with dimensions $(m \times m)$ and $(\tilde{m} \times \tilde{m})$ respectively that satisfy equations: $\Lambda_{\tilde{\mathbf{q}}} \tilde{\mathcal{M}}_{0}-\tilde{\mathcal{M}}_{0} \Lambda_{\tilde{\mathbf{r}}}^{\top}=0, \Lambda_{\mathbf{q}} \mathcal{M}_{0}-\mathcal{M}_{0} \Lambda_{\mathbf{r}}^{\top}=0$.

Proof. The proof is a direct consequence of reductions (4.2) and Proposition 2.3.

Consider some examples of the hierarchy given by (4.1) and (4.3).

1. $k=1, n=2$.

$$
\begin{aligned}
& L_{1}=\beta_{1} \partial_{\tau_{1}}-D-\mathbf{q} \mathcal{M}_{0} D^{-1} \mathbf{r}^{\top} D, \\
& M_{2}=\alpha_{2} \partial_{t_{2}}-D^{2}-v D-\tilde{\mathbf{q}} \tilde{\mathcal{M}}_{0} D^{-1} \tilde{\mathbf{r}}^{\top} D .
\end{aligned}
$$

Lax representation $\left[L_{1}, M_{2}\right]=0$ is equivalent to the following system:

$$
\begin{aligned}
& \alpha_{2} \mathbf{q}_{t_{2}}-\mathbf{q}_{x x}-v \mathbf{q}_{x}-\tilde{\mathbf{q}} \tilde{\mathcal{M}}_{0} D^{-1}\left\{\tilde{\mathbf{r}}^{\top} \mathbf{q}_{x}\right\}=\mathbf{q} \Lambda_{\mathbf{q}}, \\
& -\alpha_{2} \mathbf{r}_{t_{2}}-\mathbf{r}_{x x}+v^{\top} \mathbf{r}_{x}-\tilde{\mathbf{r}} \tilde{\mathcal{M}}_{0}^{\top} D^{-1}\left\{\tilde{\mathbf{q}}^{\top} \mathbf{r}_{x}\right\}=\mathbf{r} \Lambda_{\mathbf{r}}, \\
& \beta_{1} \tilde{\mathbf{q}}_{\tau_{1}}-\tilde{\mathbf{q}}_{x}-\mathbf{q} \mathcal{M}_{0} D^{-1}\left\{\mathbf{r}^{\top} \tilde{\mathbf{q}}_{x}\right\}=\tilde{\mathbf{q}} \Lambda_{\tilde{\mathbf{q}}}, \quad-\beta_{1} \tilde{\mathbf{r}}_{\tau_{1}}+\tilde{\mathbf{r}}_{x}-\mathbf{r} \mathcal{M}_{0}^{\top} D^{-1}\left\{\mathbf{q}^{\top} \tilde{\mathbf{r}}_{x}\right\}=\tilde{\mathbf{r}} \Lambda_{\tilde{\mathbf{r}}}, \\
& v_{x}-\beta_{1} v_{\tau_{1}}=2\left(\mathbf{q} \mathcal{M}_{0} \mathbf{r}^{\top}\right)_{x} .
\end{aligned}
$$

In case of the Hermitian conjugation reduction $\beta_{1} \in \mathbb{R}, \alpha_{2} \in i \mathbb{R}, \mathcal{M}_{0}^{*}=-\mathcal{M}_{0}, \tilde{\mathcal{M}}_{0}^{*}=\tilde{\mathcal{M}}_{0}$, $\overline{\tilde{\mathbf{r}}}=\tilde{\mathbf{q}}, \overline{\mathbf{r}}=\mathbf{q}, v=-v^{*}\left(L_{1}^{*}=-D L_{1} D^{-1}, M_{2}^{*}=D M_{2} D^{-1}\right)$ the latter equation reduces to the following one:

$$
\begin{aligned}
& \alpha_{2} \mathbf{q}_{t_{2}}-\mathbf{q}_{x x}-v \mathbf{q}_{x}-\tilde{\mathbf{q}} \tilde{\mathcal{M}}_{0} D^{-1}\left\{\tilde{\mathbf{q}}^{*} \mathbf{q}_{x}\right\}=\mathbf{q} \Lambda_{\mathbf{q}} \\
& \beta_{1} \tilde{\mathbf{q}}_{\tau_{1}}-\tilde{\mathbf{q}}_{x}-\mathbf{q} \mathcal{M}_{0} D^{-1}\left\{\mathbf{q}^{*} \tilde{\mathbf{q}}_{x}\right\}=\tilde{\mathbf{q}} \Lambda_{\tilde{\mathbf{q}}}, \quad v_{x}-\beta_{1} v_{\tau_{1}}=2\left(\mathbf{q} \mathcal{M}_{0} \mathbf{q}^{*}\right)_{x} .
\end{aligned}
$$

In case we set $\tilde{\mathbf{q}}=0, \Lambda_{\mathbf{q}}=0$ we get a matrix $(2+1)$-dimensional generalization of the ChenLee-Liu equation

$$
\alpha_{2} \mathbf{q}_{t_{2}}-\mathbf{q}_{x x}-v \mathbf{q}_{x}=0, \quad v_{x}-\beta_{1} v_{\tau_{1}}=2\left(\mathbf{q} \mathcal{M}_{0} \mathbf{q}^{*}\right)_{x}
$$

2. $k=3, n=2$.

$$
\begin{aligned}
& L_{3}=\beta_{3} \partial_{\tau_{3}}-c_{1}\left(D^{3}+w D^{2}+v D\right)-\mathbf{q} \mathcal{M}_{0} D^{-1} \mathbf{r}^{\top} D \\
& M_{2}=\alpha_{2} \partial_{t_{2}}-c_{2} D^{2}-u D-\tilde{\mathbf{q}} \tilde{\mathcal{M}}_{0} D^{-1} \tilde{\mathbf{r}}^{\top} D .
\end{aligned}
$$

Using (4.3) we get that Lax representation $\left[L_{3}, M_{2}\right]=0$ is equivalent to the following system

$$
\begin{aligned}
& 3 u_{x}-2 c_{2} w_{x}-[u, w]=0, \\
& \alpha_{2} c_{1} w_{t_{2}}-c_{1} c_{2} w_{x x}+2 c_{1} w u_{x}-c_{1} u w_{x}+3 c_{1} u_{x x} \\
& \quad+3 c_{1}\left(\tilde{\mathbf{q}} \tilde{\mathcal{M}}_{0} \tilde{\mathbf{r}}^{\top}\right)_{x}-2 c_{2} c_{1} v_{x}-c_{2}\left[\tilde{\mathbf{q}} \tilde{\mathcal{M}}_{0} \tilde{\mathbf{r}}^{\top}, w\right]+c_{1}[v, u]=0, \\
& \quad-\beta_{3} u_{\tau_{3}}+c_{1} u_{x x x}+c_{1} w u_{x x}+3 c_{1}\left(\tilde{\mathbf{q}}_{x} \tilde{\mathcal{M}}_{0} \tilde{\mathbf{r}}^{\top}\right)_{x}+2 c_{1} w \tilde{\mathbf{q}}_{x} \tilde{\mathcal{M}}_{0} \tilde{\mathbf{r}}^{\top}
\end{aligned}
$$




$$
\begin{aligned}
& \quad+c_{1} \tilde{\mathbf{q}} \tilde{\mathcal{M}}_{0} \tilde{\mathbf{r}}_{x}^{\top} w+\alpha_{2} c_{1} v_{t_{2}}-c_{1} c_{2} v_{x x}+c_{1} v u_{x}-c_{1} u v_{x}+c_{1}\left[v, \tilde{\mathbf{q}} \mathcal{M}_{0} \tilde{\mathbf{r}}^{\top}\right] \\
& \quad-2 c_{2}\left(\mathbf{q} \mathcal{M}_{0} \mathbf{r}^{\top}\right)_{x}+c_{1} w \tilde{\mathbf{q}} \tilde{\mathcal{M}}_{0} \tilde{\mathbf{r}}_{x}^{\top}+\left[\mathbf{q} \mathcal{M}_{0} \mathbf{r}^{\top}, u\right]=0, \\
& \beta_{3} \tilde{\mathbf{q}}_{\tau_{3}}-c_{1} \tilde{\mathbf{q}}_{x x x}-c_{1} w \tilde{\mathbf{q}}_{x x}-c_{3} v \tilde{\mathbf{q}}_{x}-\mathbf{q} \mathcal{M}_{0} D^{-1}\left\{\mathbf{r}^{\top} \tilde{\mathbf{q}}_{x}\right\}=\tilde{\mathbf{q}} \Lambda_{\tilde{\mathbf{q}}}, \\
& \alpha_{2} \mathbf{q}_{t_{2}}-c_{2} \mathbf{q}_{x x}-u \mathbf{q}_{x}-\tilde{\mathbf{q}} \tilde{\mathcal{M}}_{0} D^{-1}\left\{\tilde{\mathbf{r}}^{\top} \mathbf{q}_{x}\right\}=\mathbf{q} \Lambda_{\mathbf{q}}, \\
& -\beta_{3} \tilde{\mathbf{r}}_{\tau_{3}}+c_{1} \tilde{\mathbf{r}}_{x x x}-c_{1}\left(w^{\top} \tilde{\mathbf{r}}_{x}\right)_{x}+c_{1} v^{\top} \tilde{\mathbf{r}}_{x}-\mathbf{r} \mathcal{M}_{0}^{\top} D^{-1}\left\{\mathbf{q}^{\top} \tilde{\mathbf{r}}_{x}\right\}=\tilde{\mathbf{r}} \Lambda_{\tilde{\mathbf{r}}}, \\
& -\alpha_{2} \mathbf{r}_{t_{2}}-c_{2} \mathbf{r}_{x x}+u^{\top} \mathbf{r}_{x}-\tilde{\mathbf{r}} \tilde{\mathcal{M}}_{0}^{\top} D^{-1}\left\{\tilde{\mathbf{q}}^{\top} \mathbf{r}_{x}\right\}=\mathbf{r} \Lambda_{\mathbf{r}} .
\end{aligned}
$$

Set $c_{1}=c_{2}=1$ in the scalar case $(N=1)$. Eliminating variables $w$ and $v$ from the first and second equation respectively, we get

$$
\begin{aligned}
& -\beta_{3} u_{\tau_{3}}-\frac{1}{4} u_{x x x}-\frac{3}{8} u^{2} u_{x}+\frac{3}{4} \alpha_{2} u_{x} D^{-1}\left\{u_{t_{2}}\right\}+\frac{3}{4} \alpha_{2}^{2} D^{-1}\left\{u_{t_{2} t_{2}}\right\} \\
& \quad+\frac{3}{2}\left(u \tilde{\mathbf{q}} \tilde{\mathcal{M}}_{0} \tilde{\mathbf{r}}^{\top}\right)_{x}+\frac{3}{2} \alpha_{2}\left(\tilde{\mathbf{q}} \mathcal{M}_{0} \tilde{\mathbf{r}}^{\top}\right)_{t_{2}}-\frac{3}{2}\left(\tilde{\mathbf{q}} \tilde{\mathcal{M}}_{0} \tilde{\mathbf{r}}^{\top}\right)_{x x}-2\left(\mathbf{q} \mathcal{M}_{0} \mathbf{r}^{\top}\right)_{x}=0, \\
& \beta_{3} \tilde{\mathbf{q}}_{\tau_{3}}-\tilde{\mathbf{q}}_{x x x}-\frac{3}{2} u \tilde{\mathbf{q}}_{x x}-v \tilde{\mathbf{q}}_{x}-\mathbf{q} \mathcal{M}_{0} D^{-1}\left\{\mathbf{r}^{\top} \tilde{\mathbf{q}}_{x}\right\}=\tilde{\mathbf{q}} \Lambda_{\tilde{\mathbf{q}}}, \\
& \alpha_{2} \mathbf{q}_{t_{2}}-\mathbf{q}_{x x}-u \mathbf{q}_{x}-\tilde{\mathbf{q}} \tilde{\mathcal{M}}_{0} D^{-1}\left\{\tilde{\mathbf{r}}^{\top} \mathbf{q}_{x}\right\}=\mathbf{q} \Lambda_{\mathbf{q}}, \\
& -\beta_{3} \tilde{\mathbf{r}}_{\tau_{3}}+\tilde{\mathbf{r}}_{x x x}-\frac{3}{2}\left(u \tilde{\mathbf{r}}_{x}\right)_{x}+v \tilde{\mathbf{r}}_{x}-\mathbf{r} \mathcal{M}_{0}^{\top} D^{-1}\left\{\mathbf{q}^{\top} \tilde{\mathbf{r}}_{x}\right\}=\tilde{\mathbf{r}} \Lambda_{\tilde{\mathbf{r}}}, \\
& -\alpha_{2} \mathbf{r}_{t_{2}}-\mathbf{r}_{x x}+u \mathbf{r}_{x}-\tilde{\mathbf{r}} \tilde{\mathcal{M}}_{0}^{\top} D^{-1}\left\{\tilde{\mathbf{q}}^{\top} \mathbf{r}_{x}\right\}=\mathbf{r} \Lambda_{\mathbf{r}}, \\
& v=\frac{3}{4} u_{x}+\frac{3}{8} u^{2}+\frac{3}{2}\left(\tilde{\mathbf{q}} \tilde{\mathcal{M}}_{0} \tilde{\mathbf{r}}^{\top}\right)+\frac{3}{4} \alpha_{2} D^{-1}\left\{u_{t_{2}}\right\} .
\end{aligned}
$$

The latter under the Hermitian conjugation reduction $\alpha_{2} \in i \mathbb{R}, \beta_{3} \in \mathbb{R}, \mathcal{M}_{0}^{*}=-\mathcal{M}_{0}, \tilde{\mathcal{M}}_{0}=\tilde{\mathcal{M}}_{0}^{*}$, $\bar{u}=-u, \overline{\tilde{\mathbf{r}}}=\tilde{\mathbf{q}}, \overline{\mathbf{r}}=\mathbf{q}\left(L_{3}^{*}=-D L_{3} D^{-1}, M_{2}^{*}=D M_{2} D^{-1}\right)$ reads:

$$
\begin{aligned}
& -\beta_{3} u_{\tau_{3}}-\frac{1}{4} u_{x x x}-\frac{3}{8} u^{2} u_{x}+\frac{3}{4} \alpha_{2} u_{x} D^{-1}\left\{u_{t_{2}}\right\}+\frac{3}{4} \alpha_{2}^{2} D^{-1}\left\{u_{t_{2} t_{2}}\right\} \\
& \quad+\frac{3}{2}\left(u \tilde{\mathbf{q}} \tilde{\mathcal{M}}_{0} \tilde{\mathbf{q}}^{*}\right)_{x}+\frac{3}{2} \alpha_{2}\left(\tilde{\mathbf{q}} \tilde{\mathcal{M}}_{0} \tilde{\mathbf{q}}^{*}\right)_{t_{2}}-\frac{3}{2}\left(\tilde{\mathbf{q}} \tilde{\mathcal{M}}_{0} \tilde{\mathbf{q}}^{*}\right)_{x x}-2\left(\mathbf{q} \mathcal{M}_{0} \mathbf{q}^{*}\right)_{x}=0 \\
& \beta_{3} \tilde{\mathbf{q}}_{\tau_{3}}-\tilde{\mathbf{q}}_{x x x}-\frac{3}{2} u \tilde{\mathbf{q}} x x-\left(\frac{3}{4} u_{x}+\frac{3}{8} u^{2}+\frac{3}{2}\left(\tilde{\mathbf{q}} \tilde{\mathcal{M}}_{0} \tilde{\mathbf{q}}^{*}\right)+\frac{3}{4} \alpha_{2} D^{-1}\left\{u_{t_{2}}\right\}\right) \tilde{\mathbf{q}}_{x} \\
& \quad-\mathbf{q} \mathcal{M}_{0} D^{-1}\left\{\mathbf{q}^{*} \tilde{\mathbf{q}}_{x}\right\}=\tilde{\mathbf{q}} \Lambda_{\tilde{\mathbf{q}}} \\
& \alpha_{2} \mathbf{q}_{t_{2}}-\mathbf{q}_{x x}-u \mathbf{q}_{x}-\tilde{\mathbf{q}} \tilde{\mathcal{M}}_{0} D^{-1}\left\{\tilde{\mathbf{r}}^{\top} \mathbf{q}_{x}\right\}=\mathbf{q} \Lambda_{\mathbf{q}} .
\end{aligned}
$$

$\tilde{\mathbf{q}}=0, \Lambda_{\mathbf{q}}=0$ lead to the modified KPSCS of the first type

$$
\begin{aligned}
& -\beta_{3} u_{\tau_{3}}-\frac{1}{4} u_{x x x}-\frac{3}{8} u^{2} u_{x}+\frac{3}{4} \alpha_{2} u_{x} D^{-1}\left\{u_{t_{2}}\right\}+\frac{3}{4} \alpha_{2}^{2} D^{-1}\left\{u_{t_{2} t_{2}}\right\}=2\left(\mathbf{q} \mathcal{M}_{0} \mathbf{q}^{*}\right)_{x} \\
& \alpha_{2} \mathbf{q}_{t_{2}}-\mathbf{q}_{x x}-u \mathbf{q}_{x}=0 .
\end{aligned}
$$

If $\mathbf{q}=0, \tilde{\Lambda}_{\tilde{\mathbf{q}}}=0$ in (4.5) we recover the second type of the modified KPSCS

$$
\begin{aligned}
& -\beta_{3} u_{\tau_{3}}-\frac{1}{4} u_{x x x}-\frac{3}{8} u^{2} u_{x}+\frac{3}{4} \alpha_{2} u_{x} D^{-1}\left\{u_{t_{2}}\right\}+\frac{3}{4} \alpha_{2}^{2} D^{-1}\left\{u_{t_{2} t_{2}}\right\} \\
& \quad+\frac{3}{2}\left(u \tilde{\mathbf{q}} \tilde{\mathcal{M}}_{0} \tilde{\mathbf{q}}^{*}\right)_{x}+\frac{3}{2} \alpha_{2}\left(\tilde{\mathbf{q}} \tilde{\mathcal{M}}_{0} \tilde{\mathbf{q}}^{*}\right)_{t_{2}}-\frac{3}{2}\left(\tilde{\mathbf{q}} \tilde{\mathcal{M}}_{0} \tilde{\mathbf{q}}^{*}\right)_{x x}=0, \\
& \beta_{3} \tilde{\mathbf{q}}_{\tau_{3}}-\tilde{\mathbf{q}}_{x x x}-\frac{3}{2} u \tilde{\mathbf{q}}_{x x}-\left(\frac{3}{4} u_{x}+\frac{3}{8} u^{2}+\frac{3}{2}\left(\tilde{\mathbf{q}} \tilde{\mathcal{M}}_{0} \tilde{\mathbf{q}}^{*}\right)+\frac{3}{4} \alpha_{2} D^{-1}\left\{u_{t_{2}}\right\}\right) \tilde{\mathbf{q}}_{x}=0,
\end{aligned}
$$

Both types were investigated in [47] within $(2+1)$-dimensional extensions of the $k$-cmKP hierarchy. 


\subsection{Dressing via binary Darboux transformations}

In this subsection we consider dressing methods for $(2+1)$-dimensional extensions of the modified $k$-constrained KP hierarchy given by (4.1) and (4.3). First of all, we start with the matrix version of the theorem that was proven in [12].

Theorem 4.2. Let $(N \times K)$-matrix functions $\varphi$ and $\psi$ satisfy linear problems:

$$
\begin{aligned}
& L_{k}\{\varphi\}=\varphi \Lambda_{k}, L_{k}^{\tau}\{\psi\}=\psi \tilde{\Lambda}_{k}, \quad \Lambda_{k}, \tilde{\Lambda}_{k} \in \operatorname{Mat}_{K \times K}(\mathbb{C}), \\
& L_{k}=\beta_{k} \partial_{\tau_{k}}-B_{k}-\mathbf{q} \mathcal{M}_{0} D^{-1} \mathbf{r}^{\top} D, \quad B_{k}=\sum_{i=1}^{k} u_{i} D^{i} .
\end{aligned}
$$

Then the operator $L_{k}$ transformed via

$$
W_{m}:=w_{0}^{-1} W=w_{0}^{-1}\left(I-\varphi \Delta^{-1} D^{-1} \psi^{\top}\right)=I-\varphi \tilde{\Delta}^{-1} D^{-1}\left(D^{-1}\{\psi\}\right)^{\top} D,
$$

where

$$
\begin{aligned}
& w_{0}=I_{N}-\varphi \Delta^{-1} D^{-1}\left\{\psi^{\top}\right\}, \quad \tilde{\Delta}=-C+D^{-1}\left\{D^{-1}\left\{\psi^{\top}\right\} \varphi_{x}\right\}, \\
& \Delta=C+D^{-1}\left\{\psi^{\top} \varphi\right\},
\end{aligned}
$$

has the form:

$$
\begin{aligned}
& \hat{L}_{k}:=W_{m} L_{k} W_{m}^{-1}=\beta_{k} \partial_{\tau_{k}}-\hat{B}_{k}-\hat{\mathbf{q}} \mathcal{M}_{0} D^{-1} \hat{\mathbf{r}}^{\top} D+\Phi \mathcal{M}_{k} D^{-1} \Psi^{\top} D, \\
& \hat{B}_{k}=\sum_{j=1}^{k} \hat{u}_{j} D^{j}, \quad \hat{u}_{k}=u_{k}, \quad \hat{u}_{k-1}=u_{k-1}+k u_{k} w_{0}^{-1} w_{0, x}, \quad \ldots,
\end{aligned}
$$

where

$$
\begin{aligned}
& \mathcal{M}_{k}=C \Lambda_{k}-\tilde{\Lambda}_{k}^{\top} C, \quad \tilde{\Phi}=-W_{m}\{\varphi\} C^{-1}=\varphi \tilde{\Delta}^{-1}, \\
& \tilde{\Psi}=D^{-1}\left\{W_{m}^{\tau,-1}\{\psi\}\right\} C^{-1, \top}=D^{-1}\{\psi\} \Delta^{-1, \top}, \quad \hat{\mathbf{q}}=W_{m}\{\mathbf{q}\}, \hat{\mathbf{r}}=D^{-1} W_{m}^{-1, \tau} D\{\mathbf{r}\} .
\end{aligned}
$$

Proof. The proof is analogous to the proof of Theorem 2 in [12].

The following consequence of the latter theorem provides a solution generating method for the hierarchy given by (4.1) and (4.3):

Corollary 4.3. Let $(N \times K)$-matrix functions $\varphi$ and $\psi$ satisfy linear problems:

$$
\begin{aligned}
& L_{k}\{\varphi\}=\varphi \Lambda_{k, 1}, \quad L_{k}^{\tau}\{\psi\}=\psi \tilde{\Lambda}_{k, 1}, \quad \Lambda_{k, 1}, \tilde{\Lambda}_{k, 1} \in \operatorname{Mat}_{K \times K}(\mathbb{C}), \\
& M_{n}\{\varphi\}=\varphi \Lambda_{n, 2}, \quad M_{n}^{\tau}\{\psi\}=\psi \tilde{\Lambda}_{n, 2}, \quad \Lambda_{n, 2}, \tilde{\Lambda}_{n, 2} \in \operatorname{Mat}_{K \times K}(\mathbb{C})
\end{aligned}
$$

with operators $L_{k}$ and $M_{n}$ given by (4.1).

The operators $\hat{L}_{k}=W_{m} L_{k} W_{m}^{-1}$ and $\hat{M}_{n}=W_{m} M_{n} W_{m}^{-1}$ transformed via $W_{m}$ (4.6), (4.7) have the form:

$$
\begin{array}{ll}
\hat{L}_{k}:=\beta_{k} \partial_{\tau_{k}}-\hat{B}_{k}-\hat{\mathbf{q}} \mathcal{M}_{0} D^{-1} \hat{\mathbf{r}}^{\top} D+\tilde{\Phi} \mathcal{M}_{k, 1} D^{-1} \tilde{\Psi}^{\top} D, & \hat{B}_{k}=\sum_{j=1}^{k} \hat{u}_{j} D^{j}, \\
\hat{M}_{n}=\alpha_{n} \partial_{t_{n}}-\hat{A}_{n}-\hat{\mathbf{q}} \tilde{\mathcal{M}}_{0} D^{-1} \hat{\mathbf{r}}^{\top} D+\tilde{\Phi} \mathcal{M}_{n, 2} D^{-1} \tilde{\Psi}^{\top} D, & \hat{A}_{n}=\sum_{i=1}^{n} \hat{v}_{i} D^{i},
\end{array}
$$

where

$$
\begin{aligned}
& \mathcal{M}_{k, 1}=C \Lambda_{k, 1}-\tilde{\Lambda}_{k, 1}^{\top} C, \quad \mathcal{M}_{n, 2}=C \Lambda_{n, 2}-\tilde{\Lambda}_{n, 2}^{\top} C, \quad \tilde{\Phi}=-W_{m}\{\varphi\} C^{-1}=\varphi \tilde{\Delta}^{-1}, \\
& \tilde{\Psi}=D^{-1}\left\{W_{m}^{\tau,-1}\{\psi\}\right\} C^{-1, \top}=D^{-1}\{\psi\} \Delta^{-1, \top}, \quad \hat{\mathbf{q}}=W_{m}\{\mathbf{q}\}, \\
& \hat{\mathbf{r}}=D^{-1} W_{m}^{-1, \tau} D\{\mathbf{r}\}, \quad \tilde{\Delta}=-C+D^{-1}\left\{D^{-1}\left\{\psi^{\top}\right\} \varphi_{x}\right\} .
\end{aligned}
$$




\section{Conclusion}

In this work we proposed new integrable generalizations of the KP and modified KP hierarchy with self-consistent sources. The obtained hierarchies of nonlinear equations include, in particular, matrix integrable system that contains as special cases two types of the matrix KP equation with self-consistent sources (KPSCS) and its modified version. They also cover new generalizations of the $N$-wave problem and the DS-III system. Under reductions (2.11) imposed on the obtained hierarchies one recovers $(2+1)$-BD $k$-cKP hierarchy. The latter contains $\left(t_{A}, \tau_{B}\right)$ - and $\left(\gamma_{A}, \sigma_{B}\right)$-matrix KP hierarchies [36, 37] (see [11] for details).

Remark 5.1. It should be pointed out that in the scalar case $(N=1)$ Lax pairs (4.1) admit the following reduction:

$$
\begin{aligned}
& \mathbf{q}=\left(\mathbf{q}_{1}, \mathbf{q}_{2},-\mathbf{q}_{2} \mathcal{M}_{0} \mathbf{r}_{2}^{\top}-D^{-1}\{u\}, 1\right), \quad \mathbf{r}=\left(\mathbf{r}_{1}, D^{-1}\left\{\mathbf{r}_{2}\right\}, 1, D^{-1}\{u\}\right), \\
& \tilde{\mathbf{q}}=\left(\tilde{\mathbf{q}}_{1}, \tilde{\mathbf{q}}_{2},-\tilde{\mathbf{q}}_{2} \tilde{\mathcal{M}}_{1} \tilde{\mathbf{r}}_{2}^{\top}-D^{-1}\{\tilde{u}\}, 1, \mathbf{q}_{1}[0], \mathbf{q}_{1}[1], \ldots, \mathbf{q}_{1}[l]\right), \quad \mathbf{q}_{1}[j]:=L^{j}\left\{\mathbf{q}_{1}\right\}, \\
& \tilde{\mathbf{r}}=\left(\tilde{\mathbf{r}}_{1}, \tilde{\mathbf{r}}_{2}, 1, D^{-1}\{\tilde{u}\}, \mathbf{r}_{1}[l], \mathbf{r}_{1}[l-1], \ldots, \mathbf{r}_{1}[0]\right), \quad \mathbf{r}_{1}[j]:=\left(L^{\tau}\right)^{j}\left\{\mathbf{r}_{1}\right\}, \\
& \mathcal{M}_{0}=\operatorname{diag}\left(\mathcal{M}_{1}, \mathcal{M}_{1}, 1,1\right), \quad \tilde{\mathcal{M}}_{0}=\operatorname{diag}\left(\tilde{\mathcal{M}}_{1}, \tilde{\mathcal{M}}_{1}, 1,1, I_{l+1} \otimes \mathcal{M}_{1}\right),
\end{aligned}
$$

where $\mathbf{q}_{j}, \mathbf{r}_{j}$ and $\tilde{\mathbf{q}}_{j}, \tilde{\mathbf{r}}_{j}, j=1,2$, are vectors of functions of dimensions $\left(1 \times m_{0}\right)$ and $\left(1 \times \tilde{m}_{0}\right)$ respectively. $\mathcal{M}_{1}$ and $\tilde{\mathcal{M}}_{1}$ are square matrices of dimensions $m_{0}$ and $\tilde{m}_{0} \cdot I_{l+1} \otimes \mathcal{M}_{1}$ denotes the tensor product of the identity matrix $I_{l+1}$ and $\mathcal{M}_{1}$.

It leads to the following family of integro-differential operators in (4.1)

$$
\begin{aligned}
L_{k}= & \beta_{k} \partial_{\tau_{k}}-B_{k}-\mathbf{q}_{1} \mathcal{M}_{1} D^{-1} \mathbf{r}_{1}^{\top} D+\mathbf{q}_{2} \mathcal{M}_{1} D^{-1} \mathbf{r}_{2}^{\top}+D^{-1} u, \\
M_{n}= & \alpha_{n} \partial_{t_{n}}-A_{n}-\tilde{\mathbf{q}}_{1} \tilde{\mathcal{M}}_{1} D^{-1} \tilde{\mathbf{r}}_{1}^{\top} D+\tilde{\mathbf{q}}_{2} \tilde{\mathcal{M}}_{1} D^{-1} \tilde{\mathbf{r}}_{2}^{\top}+D^{-1} \tilde{u} \\
& -c_{l} \sum_{j=0}^{l} \mathbf{q}_{1}[j] \mathcal{M}_{0} D^{-1} \mathbf{r}_{1}^{\top}[l-j] D .
\end{aligned}
$$

Lax equation $\left[L_{k}, M_{n}\right]=0$ involving the latter operators should lead (under additional reductions) to $(2+1)$-dimensional generalizations of the corresponding integrable systems that were obtained in [12]. In particular it concerns systems that extend $\mathrm{KdV}, \mathrm{mKdV}$ and Kaup-Broer equations.

In this paper we also elaborated solution generating methods for the proposed hierarchies (2.2), (2.6) and (4.1), (4.3) respectively via DTs and BDTs.

The latter involve fixed solutions of linear problems and an arbitrary seed (initial) solution of the corresponding integrable system. Exact solutions of equations with self-consistent sources (complexitons, negatons, positons) and the underlying hierarchies were studied in [36, 49, 85]. One of the problems for future interest consists in looking for the corresponding analogues of these solutions in the obtained generalizations. The same question concerns lumps and rogue wave solutions that were investigated in several integrable systems recently $[3,20,21,22,23$, $24,62,81]$

It is also known that inverse scattering and spectral methods $[1,7,41,57]$ were applied to generate solutions of equations with self-consistent sources $[6,27,46]$. An extension of these methods to the obtained hierarchies and comparison with results that can be provided by BDTs (e.g., following [67]) presents an interest for us.

The search for the corresponding discrete counterparts of the constructed hierarchies is another problem for future investigation. The latter is expected to contain the discrete KP equation with self-consistent sources $[19,35]$. One of the possible ways to solve the problem consists in looking for the formulation of the corresponding continuous hierarchy within a framework of bidifferential calculus. The latter framework provides better possibilities to search for the discrete counterparts of the corresponding continuous systems (see, e.g., [17]). 


\section{Acknowledgements}

The authors are grateful to Professors Folkert Müller-Hoissen and Maxim Pavlov for fruitful discussions and useful advice in preparation of this paper. The authors also wish to express their gratitude to the referees for their valuable comments and suggestions. O. Chvartatskyi has been supported via the Alexander von Humboldt foundation. Yu.M. Sydorenko is grateful to the Ministry of Education, Science, Youth and Sports of Ukraine for partial financial support (Research Grant MA-107F).

\section{References}

[1] Ablowitz M.J., Clarkson P.A., Solitons, nonlinear evolution equations and inverse scattering, London Mathematical Society Lecture Note Series, Vol. 149, Cambridge University Press, Cambridge, 1991.

[2] Ablowitz M.J., Haberman R., Nonlinear evolution equations-two and three dimensions, Phys. Rev. Lett. 35 (1975), 1185-1188.

[3] Akhmediev N., Soto-Crespo J.M., Ankiewicz A., Extreme waves that appear from nowhere: on the nature of rogue waves, Phys. Lett. A 373 (2009), 2137-2145.

[4] Aratyn H., Nissimov E., Pacheva S., Constrained KP hierarchies: additional symmetries, DarbouxBäcklund solutions and relations to multi-matrix models, Internat. J. Modern Phys. A 12 (1997), 1265-1340, hep-th/9607234.

[5] Blackmore D., Prykarpatsky A.K., Samoylenko V.H., Nonlinear dynamical systems of mathematical physics. Spectral and symplectic integrability analysis, World Sci. Publ. Co. Pte. Ltd., Hackensack, NJ, 2011.

[6] Bondarenko N., Freiling G., Urazboev G., Integration of the matrix KdV equation with self-consistent source, Chaos Solitons Fractals 49 (2013), 21-27.

[7] Calogero F., Degasperis A., Spectral transform and solitons. Vol. I. Tools to solve and investigate nonlinear evolution equations, Studies in Mathematics and its Applications, Vol. 13, North-Holland Publishing Co., Amsterdam - New York, 1982.

[8] Cheng Y., Constraints of the Kadomtsev-Petviashvili hierarchy, J. Math. Phys. 33 (1992), 3774-3782.

[9] Cheng Y., Li Y.S., The constraint of the Kadomtsev-Petviashvili equation and its special solutions, Phys. Lett. A 157 (1991), 22-26.

[10] Cheng Y., Li Y.S., Constraints of the (2+1)-dimensional integrable soliton systems, J. Phys. A: Math. Gen. 25 (1992), 419-431.

[11] Chvartatskyi O.I., Sydorenko Yu.M., A new bidirectional generalization of $(2+1)$-dimensional matrix $k$ constrained Kadomtsev-Petviashvili hierarchy, J. Math. Phys. 54 (2013), 113508, 22 pages, arXiv:1303.6510.

[12] Chvartatskyi O.I., Sydorenko Yu.M., Additional reductions in the $k$-constrained modified KP hierarchy, Nonlinear Oscil. 17 (2014), 419-436, arXiv:1303.6509.

[13] Dickey L.A., Soliton equations and Hamiltonian systems, Advanced Series in Mathematical Physics, Vol. 26, 2nd ed., World Sci. Publ. Co., Inc., River Edge, NJ, 2003.

[14] Dimakis A., Müller-Hoissen F., Exploration of the extended ncKP hierarchy, J. Phys. A: Math. Gen. 37 (2004), 10899-10930, hep-th/0406112.

[15] Dimakis A., Müller-Hoissen F., An algebraic scheme associated with the non-commutative KP hierarchy and some of its extensions, J. Phys. A: Math. Gen. 38 (2005), 5453-5505, nlin.SI/0501003.

[16] Dimakis A., Müller-Hoissen F., Bidifferential calculus approach to AKNS hierarchies and their solutions, SIGMA 6 (2010), 055, 27 pages, arXiv:1004.1627.

[17] Dimakis A., Müller-Hoissen F., Solutions of matrix NLS systems and their discretizations: a unified treatment, Inverse Problems 26 (2010), 095007, 55 pages, arXiv:1001.0133.

[18] Dimakis A., Müller-Hoissen F., Binary Darboux transformations in bidifferential calculus and integrable reductions of vacuum Einstein equations, SIGMA 9 (2013), 009, 31 pages, arXiv:1207.1308.

[19] Doliwa A., Lin R., Discrete KP equation with self-consistent sources, Phys. Lett A 378 (2014), 1925-1931, arXiv:1310.4636.

[20] Dubard P., Gaillard P., Klein C., Matveev V.B., On multi-rogue wave solutions of the NLS equation and positon solutions of the KdV equation, Eur. Phys. J. Special Topics 185 (2010), 247-258. 
[21] Dubard P., Matveev V.B., Multi-rogue waves solutions: from the NLS to the KP-I equation, Nonlinearity 26 (2013), R93-R125.

[22] Dubrovsky V.G., Formusatik I.B., The construction of exact rational solutions with constant asymptotic values at infinity of two-dimensional NVN integrable nonlinear evolution equations via the $\bar{\partial}$-dressing method, J. Phys. A: Math. Gen. 34 (2001), 1837-1851.

[23] Dubrovsky V.G., Formusatik I.B., New lumps of Veselov-Novikov integrable nonlinear equation and new exact rational potentials of two-dimensional stationary Schrödinger equation via $\bar{\partial}$-dressing method, Phys. Lett. A 313 (2003), 68-76.

[24] Dubrovsky V.G., Gramolin A.V., Gauge-invariant description of some $(2+1)$-dimensional integrable nonlinear evolution equations, J. Phys. A: Math. Theor. 41 (2008), 275208, 14 pages.

[25] Enriquez B., Orlov A.Yu., Rubtsov V.N., Dispersionful analogues of Benney's equations and $N$-wave systems, Inverse Problems 12 (1996), 241-250, solv-int/9510002.

[26] Fokas A.S., On the simplest integrable equation in 2+ 1, Inverse Problems 10 (1994), L19-L22.

[27] Gerdjikov V.S., Grahovski G.G., Ivanov R.I., On the (non)-integrability of KdV hierarchy with self-consistent sources, Comm. Pure Appl. Anal. 11 (2012), 1439-1452, arXiv:1109.4543.

[28] Gerdjikov V.S., Vilasi G., Yanovski A.B., Integrable Hamiltonian hierarchies. Spectral and geometric methods, Lecture Notes in Physics, Vol. 748, Springer-Verlag, Berlin, 2008.

[29] Gilson C.R., Macfarlane S.R., Dromion solutions of noncommutative Davey-Stewartson equations, J. Phys. A: Math. Theor. 42 (2009), 235202, 20 pages, arXiv:0901.4918.

[30] Gilson C.R., Nimmo J.J.C., On a direct approach to quasideterminant solutions of a noncommutative KP equation, J. Phys. A: Math. Theor. 40 (2007), 3839-3850, nlin.SI/0701027.

[31] Golenia J., Hentosh O.Ye., Prykarpatsky A.K., Integrable three-dimensional coupled nonlinear dynamical systems related to centrally extended operator Lie algebras and their Lax type three-linearization, Cent. Eur. J. Math. 5 (2007), 84-104.

[32] Hamanaka M., Toda K., Towards noncommutative integrable systems, Phys. Lett. A 316 (2003), 77-83, hep-th/0309265.

[33] Helminck G.F., van de Leur J.W., An analytic description of the vector constrained KP hierarchy, Comm. Math. Phys. 193 (1998), 627-641, solv-int/9706004.

[34] Hentosh O., Prytula M., Prykarpatsky A., Differential-geometric and Lie-algebraic foundations of investigating nonlinear dynamical systems on functional manifolds, 2nd ed., Lviv University Publ., Lviv, 2006.

[35] Hu X.-B., Wang H.-Y., Construction of dKP and BKP equations with self-consistent sources, Inverse Problems 22 (2006), 1903-1920.

[36] Huang Y., Liu X., Yao Y., Zeng Y., A new extended matrix KP hierarchy and its solutions, Theoret. and Math. Phys. 167 (2011), 590-605, arXiv:1011.4430.

[37] Huang Y.H., Yao Y.Q., Zeng Y.B., A new $\left(\gamma_{A}, \sigma_{B}\right)$-matrix KP hierarchy and its solutions, Commun. Theor. Phys. 57 (2012), 515-522, arXiv:1208.4422.

[38] Ismailov M.I., Inverse nonstationary scattering for the linear system of the 3-wave interaction problem in the case of two incident waves with the same velocity, Wave Motion 47 (2010), 205-216.

[39] Ismailov M.I., Integration of nonlinear system of four waves with two velocities in $(2+1)$ dimensions by the inverse scattering transform method, J. Math. Phys. 52 (2011), 033504, 9 pages.

[40] Konopelchenko B., Sidorenko J., Strampp W., $(1+1)$-dimensional integrable systems as symmetry constraints of (2+1)-dimensional systems, Phys. Lett. A 157 (1991), 17-21.

[41] Konopelchenko B.G., Introduction to multidimensional integrable equations. The inverse spectral transform in $2+1$ dimensions, Plenum Press, New York, 1992.

[42] Krichever I.M., General rational reductions of the Kadomtsev-Petviashvili hierarchy and their symmetries, Funct. Anal. Appl. 29 (1995), 75-80.

[43] Kundu A., Strampp W., Oevel W., Gauge transformations of constrained KP flows: new integrable hierarchies, J. Math. Phys. 36 (1995), 2972-2984.

[44] Lechtenfeld O., Mazzanti L., Penati S., Popov A.D., Tamassia L., Integrable noncommutative sine-Gordon model, Nuclear Phys. B 705 (2005), 477-503, hep-th/0406065.

[45] Lin R., Yao H., Zeng Y., Restricted flows and the soliton equation with self-consistent sources, SIGMA 2 (2006), 096, 8 pages, nlin.SI/0701003. 
[46] Lin R., Zeng Y., Ma W.-X., Solving the KdV hierarchy with self-consistent sources by inverse scattering method, Phys. A 291 (2001), 287-298.

[47] Liu X., Lin R., Jin B., Zeng Y., A generalized dressing approach for solving the extended KP and the extended mKP hierarchy, J. Math. Phys. 50 (2009), 053506, 14 pages, arXiv:0905.1402.

[48] Liu X., Zeng Y., Lin R., A new extended KP hierarchy, Phys. Lett. A 372 (2008), 3819-3823, arXiv:0710.4015.

[49] Ma W.-X., Complexiton solutions of the Korteweg-de Vries equation with self-consistent sources, Chaos Solitons Fractals 26 (2005), 1453-1458.

[50] Matveev V.B., Darboux transformation and explicit solutions of the Kadomtcev-Petviaschvily equation, depending on functional parameters, Lett. Math. Phys. 3 (1979), 213-216.

[51] Matveev V.B., Salle M.A., Darboux transformations and solitons, Springer Series in Nonlinear Dynamics, Springer-Verlag, Berlin, 1991.

[52] Mel'nikov V.K., Integration method of the Korteweg-de Vries equation with a self-consistent source, Phys. Lett. A 133 (1988), 493-496.

[53] Mel'nikov V.K., Interaction of solitary waves in the system described by the Kadomtsev-Petviashvili equation with a self-consistent source, Comm. Math. Phys. 126 (1989), 201-215.

[54] Mitropol's'kii Yu.O., Samoilenko V.G., Sidorenko Yu.M., A spatial two-dimensional generalization of the Kadomtsev-Petviashvili hierarchy with nonlocal constraints, Dopov. Nats. Akad. Nauk Ukr. (1999), no. 9, 19-23.

[55] Miwa T., Jimbo M., Date E., Solitons. Differential equations, symmetries and infinite-dimensional algebras, Cambridge Tracts in Mathematics, Vol. 135, Cambridge University Press, Cambridge, 2000.

[56] Nimmo J.J.C., Darboux transformations from reductions of the KP hierarchy, in Nonlinear Evolution Equations \& Dynamical Systems: NEEDS '94 (Los Alamos, NM), World Sci. Publ., River Edge, NJ, 1995, 168-177, solv-int/9410001.

[57] Novikov S., Manakov S.V., Pitaevskiŭ L.P., Zakharov V.E., Theory of solitons. The inverse scattering method, Contemporary Soviet Mathematics, Consultants Bureau, New York, 1984.

[58] Oevel W., Darboux theorems and Wronskian formulas for integrable systems. I. Constrained KP flows, Phys. A 195 (1993), 533-576.

[59] Oevel W., Carillo S., Squared eigenfunction symmetries for soliton equations. I, J. Math. Anal. Appl. 217 (1998), 161-178, 179-199.

[60] Oevel W., Strampp W., Wronskian solutions of the constrained Kadomtsev-Petviashvili hierarchy, J. Math. Phys. 37 (1996), 6213-6219.

[61] Ohta Y., Satsuma J., Takahashi D., Tokihiro T., An elementary introduction to Sato theory, Progr. Theoret. Phys. Suppl. (1988), 210-241.

[62] Ohta Y., Yang J., Dynamics of rogue waves in the Davey-Stewartson II equation, J. Phys. A: Math. Theor. 46 (2013), 105202, 19 pages, arXiv:1206.2548.

[63] Orlov A.Yu., Symmetries for unifying different soliton systems into a single integrable hierarchy, Preprint IINS/Oce-04/03, 1991.

[64] Orlov A.Yu., Vertex operator, $\bar{\partial}$-problem, symmetries, variational identities and Hamiltonian formalism for $2+1$ integrable systems, in Plasma Theory and Nonlinear and Turbulent Processes in Physics, Vols. 1, 2 (Kiev, 1987), Editors V.G. Bar'yakhtar, V.M. Chernousenko, N.S. Erokhin, A.G. Sitenko, V.E. Zakharov, World Sci. Publishing, Singapore, 1988, 116-134.

[65] Orlov A.Yu., Volterra operator algebra for zero curvature representation. Universality of KP, in Nonlinear Processes in Physics, Springer Series in Nonlinear Dynamics, Springer, Berlin - Heidelberg, 1993, $126-131$.

[66] Orlov A.Yu., Rauch-Wojciechowski S., Dressing method, Darboux transformation and generalized restricted flows for the KdV hierarchy, Phys. D 69 (1993), 77-84.

[67] Pochinaiko M.D., Sidorenko Yu.M., Construction of scattering operators by the method of binary Darboux transformations, Ukr. Math. J. 58 (2006), 1238-1260.

[68] Prikarpatsky Ya.A., The structure of Lax integrable flows on nonlocal manifolds: dynamical systems with sources, J. Math. Sci. 96 (1999), 3030-3037.

[69] Prykarpatsky A., Samuliak R., Blackmore D., Strampp W., Sydorenko Yu., Some remarks on Lagrangian and Hamiltonian formalism, related to infinitedimensional dynamical systems with symmetries, Cond. Matt. Phys. 6 (1995), no. 6, 79-104. 
[70] Prykarpatsky A.K., Blackmore D.L., Bogolyubov Jr. N.N., The Lie-algebraic structures and integrability of differential and differential-difference nonlineair integrable systems, Preprint, The Abdus Salam International Centre for Theoretical Physics, Miramare-Trieste, 2007.

[71] Sakhnovich A.L., Dressing procedure for solutions of nonlinear equations and the method of operator identities, Inverse Problems 10 (1994), 699-710.

[72] Sakhnovich A.L., Matrix Kadomtsev-Petviashvili equation: matrix identities and explicit non-singular solutions, J. Phys. A: Math. Gen. 36 (2003), 5023-5033.

[73] Samoilenko A.M., Samoilenko V.G., Sidorenko Yu.M., Hierarchy of the Kadomtsev-Petviashvili equations with nonlocal constraints: higher-dimensional generalizations and exact solutions of reduced systems, Ukr. Math. J. 51 (1999), 86-106.

[74] Sato M., Sato Y., Soliton equations as dynamical systems on infinite-dimensional Grassmann manifold, in Nonlinear Partial Differential Equations in Applied Science (Tokyo, 1982), North-Holland Math. Stud., Vol. 81, North-Holland, Amsterdam, 1983, 259-271.

[75] Schiebold C., A non-abelian nonlinear Schrödinger equation and countable superposition of solitons, J. Gen. Lie Theory Appl. 2 (2008), 245-250.

[76] Shabat A.B., Zakharov V.E., A scheme for integrating the nonlinear equations of mathematical physics by the method of the inverse scattering problem. I, Funct. Anal. Appl. 11 (1977), 226-235.

[77] Shabat A.B., Zakharov V.E., Integration of the nonlinear equations of mathematical physics by the method of the inverse scattering problem. II, Funct. Anal. Appl. 13 (1979), 166-174.

[78] Shchesnovich V.S., Doktorov E.V., Modified Manakov system with self-consistent source, Phys. Lett. A 213 (1996), 23-31.

[79] Sydorenko Yu., Generalized binary Darboux-like theorem for constrained Kadomtsev-Petviashvili (cKP) flows, in Proceedinds of Fifth International Conference "Symmetry in Nonlinear Mathematical Physics" (June 23-29, 2003, Kyiv), Proceedings of Institute of Mathematics, Kyiv, Vol. 50, Part 1, Editors A.G. Nikitin, V.M. Boyko, R.O. Popovych, I.A. Yehorchenko, Institute of Mathematics, Kyiv, 2004, 470-477.

[80] Sydorenko Yu., Chvartatskyi O., Binary transformations of the spatially two-dimensional operators and Lax equations, Visn. Kyiv Shevchenko Univ.: Mech. Math. 22 (2009), 32-35.

[81] Tao Y., He J., Multisolitons, breathers, and rogue waves for the Hirota equation generated by the Darboux transformation, Phys. Rev. E 85 (2012), 026601, 8 pages.

[82] Willox R., Loris I., Gilson C.R., Binary Darboux transformations for constrained KP hierarchies, Inverse Problems 13 (1997), 849-865.

[83] Xiao T., Zeng Y., Generalized Darboux transformations for the KP equation with self-consistent sources, J. Phys. A: Math. Gen. 37 (2004), 7143-7162, nlin.SI/0412070.

[84] Zakharov V.E., Manakov S.V., Resonant interaction of wave packets in nonlinear media, JETP Lett. 18 (1973), 243-245.

[85] Zeng Y., Shao Y., Xue W., Negaton and positon solutions of the soliton equation with self-consistent sources, J. Phys. A: Math. Gen. 36 (2003), 5035-5043, nlin.SI/0304030. 